\title{
The stochastic acceleration problem in two dimensions
}

\author{
Tomasz Komorowski * $\quad$ Lenya Ryzhik ${ }^{\dagger}$
}

November 5, 2018

\begin{abstract}
We consider the motion of a particle in a two-dimensional spatially homogeneous mixing potential and show that its momentum converges to the Brownian motion on a circle. This complements the limit theorem of Kesten and Papanicolaou [4 proved in dimensions $d \geq 3$.
\end{abstract}

\section{Introduction}

The momentum of a particle moving in a weakly random Hamiltonian field approaches in the long time limit the Brownian motion on the level set of the Hamiltonian in the momentum space. The position of the particle follows the trajectory generated by this momentum process. This limit has been first investigated rigorously by Kesten and Papanicolaou in 4 in dimension $d \geq 3$. More precisely, they have considered a Hamiltonian of the form

$$
\mathcal{H}(\mathbf{x}, \mathbf{v})=\frac{v^{2}}{2}+\sqrt{\delta} H(\mathbf{x}), \quad \mathbf{x} \in \mathbb{R}^{d}, \mathbf{v} \in \mathbb{R}^{d}, \quad v=|\mathbf{v}|
$$

with a spatially homogeneous and mixing random field $H(\mathbf{x})$, and $0<\delta \ll 1$. The corresponding particle trajectories are

$$
\frac{d X}{d t}=V, \quad \frac{d V}{d t}=-\sqrt{\delta} \nabla H(X), \quad X(0)=0, \quad V(0)=\mathbf{v}_{0} .
$$

As the random potential is weak, its effect becomes appreciable over large times - of the order $T \sim O(1 / \delta)$. Accordingly, we introduce the re-scaled process $X^{\delta}(t)=\delta X(t / \delta), V^{\delta}(t)=V(t / \delta)$ that satisfies

$$
\frac{d X^{\delta}}{d t}=V^{\delta}, \quad \frac{d V^{\delta}}{d t}=-\frac{1}{\sqrt{\delta}} \nabla H\left(\frac{X^{\delta}}{\delta}\right), \quad X^{\delta}(0)=0, \quad V^{\delta}(0)=\mathbf{v}_{0}
$$

Kesten and Papanicolaou have shown that the process $V^{\delta}(t)$ converges in law as $\delta \downarrow 0$ to a Brownian motion $V(t)$ on the sphere $\mathbb{S}_{v_{0}}^{d-1}=\left\{|V|=v_{0}\right\} \subset \mathbb{R}^{d}$. The process $X^{\delta}(t)$ converges (also in law) to $X(t)$, the time integral of $V(t)$ :

$$
X(t)=\int_{0}^{t} V(s) d s
$$

*Institute of Mathematics, UMCS, pl. Marii Curie Skłodowskiej 1, 20-031 Lublin, Poland; e-mail: komorow@hektor.umcs.lublin.pl

${ }^{\dagger}$ Department of Mathematics, University of Chicago, Chicago, IL 60637, USA; e-mail: ryzhik@math.uchicago.edu 
Later, Dürr, Goldstein and Lebowitz have considered the two-dimensional case [2] with a potential $H(\mathbf{x})$ of the form

$$
H(\mathbf{x})=\sum_{j} V\left(\mathbf{x}-\mathbf{r}_{j}\right)
$$

Here $\mathbf{r}_{j}$ are the locations of randomly distributed Poisson scatterers and $V$ is a compactly supported sufficiently smooth potential. They used a martingale technique to establish a result similar to that of Kesten and Papanicolaou in this case.

The goal of the present paper is to prove the diffusive limit in the general two-dimensional setting with the same assumptions on the random potential as in the original paper of Kesten and Papanicolaou. We recall that their proof was based on the following method. The main difficulty in obtaining the limit is that the random potential is time-independent, hence the time increments of $X^{\delta}(t)$ may be correlated: this happens when the trajectory comes close to its own past. To handle this issue one modifies the trajectories of the Hamiltonian system in such a way that the modified system has a better chance of being Markovian in time. The modification guarantees two properties: (i) the new trajectories will always go away from the regions of the physical space that they have just visited, and (ii) self-intersections do not lead to "gaining information about the past". The former is achieved by keeping momenta aligned locally in time, and the second by making the trajectory a straight line during the time of a self-intersection. This ensures that the modified process always sees "a new randomness" because of the spatial mixing properties of the random potential. Hence, the increments of the momentum variations are nearly independent and in the limit the modified momentum process becomes a diffusion. Next, one observes that the limit diffusion in dimensions $d \geq 3$ does stay away from its past. Hence, so does the modified trajectory process before the limit $\delta \downarrow 0$ as it is close to the aforementioned diffusion. The last step is to observe that until the trajectory comes close to its past no modification has to be made - the original and modified processes coincide. But we have shown that the modified process does not approach its past - therefore, neither does the original process as they are one and the same until the self-intersection. Hence, the process without any modification is also close to the limit diffusion, simply because the modifications were never actually made. This finishes the original proof of [4. We mention that recently we have been able to modify this method in [5] to control the particle behavior on a longer time scale and show that then the spatial component itself converges to a Brownian motion in $\mathbb{R}^{d}$.

The proof of [4] breaks down in two dimensions simply because the limit process does intersect itself - this means that so does the process before the limit and the near Markovianity of the original trajectories of the Hamiltonian system is seemingly destroyed. However, intuitively, there is some room even in two dimensions - one should avoid not all self-intersections but rather only non-transversal self-intersections as these cause the path to follow its past for a long time creating strong correlations with the past. Moreover, a non-transversal self-intersection in the physical twodimensional space is a self-intersection of the full $(X(t), V(t))$-trajectory in the three-dimensional (two spatial dimensions plus the momentum direction) phase space. The expected joint limit process $(X(t), V(t))-V(t)$ is a Brownian motion on the circle and $X(t)$ is its time integral - does not intersect itself. This allows us to use the same strategy as in [4] to push the proof through in two dimensions. The main technical difficulty and novelty of this paper is in the aforementioned control of non-transversal self-intersections of the trajectories and in the proof that this weaker constraint suffices to establish the limit.

The one-dimensional case is very different from $d \geq 2$ - see [9] for a recent discussion of this problem.

The paper is organized as follows. Section 2 contains the assumptions on the random medium and the formulation of the main result, Theorem 2.1. We introduce and study the modified dynamics in Section 3. Section 4 contains the proof of Proposition 3.5] which is the main technical estimate 
that shows that the modified process with the cut-offs is close to the momentum diffusion. The cut-offs are removed in Section [5] where Theorem [2.1 is finally proved. The appendix contains the proofs of some elementary geometric properties of trajectories.

Acknowledgment. The research of TK was partially supported by KBN grant 2PO3A03123. The work of LR was partially supported by an ONR grant N00014-02-1-0089, NSF grant DMS0203537 and an Alfred P. Sloan Fellowship.

\section{Preliminaries and the main result}

\subsection{The notation}

We begin with fixing the notation. We denote by $\mathbb{R}_{*}^{2}:=\mathbb{R}^{2} \backslash\{\mathbf{0}\}$ the range of momenta (we will assume that the initial momentum is different from zero) and by $\mathbb{R}_{*}^{4}:=\mathbb{R}^{2} \times \mathbb{R}_{*}^{2}$ the full phase space. Also $\mathbb{S}_{R}(\mathbf{x})\left(\mathbb{D}_{R}(\mathbf{x})\right)$ shall stand for a circle (open disk) of radius $R>0$ centered at $\mathbf{x}$. We shall drop writing either $\mathbf{x}$, or $R$ in the notation of the sphere (ball) in the particular cases when either $\mathbf{x}=0$, or $R=1$. For a fixed $M>10$ we define the spherical shell $A(M):=\left\{\mathbf{v} \in \mathbb{R}_{*}^{2}: M^{-1} \leq|\mathbf{v}| \leq M\right\}$ in the momentum space, and the corresponding bundle $\mathcal{A}(M):=\mathbb{R}^{2} \times A(M)$ in the whole phase space. Given a vector $\mathbf{v} \in \mathbb{R}_{*}^{2}$ we denote by $\hat{\mathbf{v}}:=\mathbf{v} /|\mathbf{v}| \in \mathbb{S}$ the unit vector in the direction of $\mathbf{v}$. For any set $A$ we shall denote by $A^{c}$ its complement.

For any non-negative integers $p, q, r$, positive times $T>T_{*} \geq 0$ and a function $G:\left[T_{*}, T\right] \times \mathbb{R}_{*}^{4} \rightarrow$ $\mathbb{R}$ that has $p, q$ and $r$ derivatives in the respective variables we define

$$
\|G\|_{p, q, r}^{\left[T_{*}, T\right]}:=\sum \sup _{(t, \mathbf{x}, \mathbf{v}) \in\left[T_{*}, T\right] \times \mathbb{R}_{*}^{4}}\left|\partial_{t}^{\alpha} \partial_{\mathbf{x}}^{\beta} \partial_{\mathbf{v}}^{\gamma} G(t, \mathbf{x}, \mathbf{v})\right| .
$$

The summation range covers all integers $0 \leq \alpha \leq p$ and all integer valued multi-indices $|\beta| \leq q$ and $|\gamma| \leq r$. We also define

$$
\|G\|_{p, q, r}=\sup _{n \geq 1}\|G\|_{p, q, r}^{[0, n]}
$$

and denote by $C_{b}^{p, q, r}\left([0,+\infty) \times \mathbb{R}_{*}^{4}\right)$ the space of all functions $G$ with $\|G\|_{p, q, r}<+\infty$. We shall also consider spaces of bounded and a suitable number of times continuously differentiable functions $C_{b}^{p, q}\left(\mathbb{R}_{*}^{4}\right)$ and $C_{b}^{p}\left(\mathbb{R}_{*}^{2}\right)$ with the respective norms $\|\cdot\|_{p, q}$ and $\|\cdot\|_{p}$.

We use the shorthand notation $\mathcal{C}:=C\left([0,+\infty) ; \mathbb{R}_{*}^{4}\right)$ for the space of continuous trajectories in the $(X, V)$-space. Let us define $(X(t), V(t)): \mathcal{C} \rightarrow \mathbb{R}_{*}^{4}$ as the canonical mapping

$$
(X(t ; \pi), V(t ; \pi)):=\pi(t), \quad \pi \in \mathcal{C} .
$$

Let also $\theta_{s}(\pi)(\cdot):=\pi(\cdot+s)$ be the standard shift transformation by $s \geq 0$.

For any $u \leq v$ denote by $\mathcal{M}_{u}^{v}$ the $\sigma$-algebra of subsets of $\mathcal{C}$ generated by $(X(t), V(t)), t \in[u, v]$. We write $\mathcal{M}^{v}:=\mathcal{M}_{0}^{v}$ and $\mathcal{M}$ for the $\sigma$ algebra of Borel subsets of $\mathcal{C}$. It coincides with the smallest $\sigma$-algebra that contains all $\mathcal{M}^{v}, v \geq 0$.

\subsection{The random medium}

Now, we describe the class of random potentials $H(\mathbf{x})$ that we consider. Let $(\Omega, \Sigma, \mathbb{P})$ be a probability space, and let $\mathbb{E}$ denote the expectation with respect to $\mathbb{P}$. The function $H: \mathbb{R}^{2} \times \Omega \rightarrow \mathbb{R}$ is a random field that is measurable and strictly stationary. This means that for any shift $\mathbf{x} \in \mathbb{R}^{2}$, and a collection of points $\mathbf{x}_{1}, \ldots, \mathbf{x}_{n} \in \mathbb{R}^{2}$ the laws of $\left(H\left(\mathbf{x}_{1}+\mathbf{x}\right), \ldots, H\left(\mathbf{x}_{n}+\mathbf{x}\right)\right)$ and $\left(H\left(\mathbf{x}_{1}\right), \ldots, H\left(\mathbf{x}_{n}\right)\right)$ 
are identical. In addition, we assume that $\mathbb{E} H(\mathbf{0})=0$, the realizations of $H(\mathbf{x})$ are $\mathbb{P}$-a.s. $C^{2}$-smooth in $\mathbf{x} \in \mathbb{R}^{2}$ and satisfy

$$
D_{i}:=\max _{|\alpha|=i} \operatorname{ess}_{(\mathbf{x} ; \omega) \in \mathbb{R}^{2} \times \Omega}\left|\partial_{\mathbf{x}}^{\alpha} H(\mathbf{x} ; \omega)\right|<+\infty, \quad i=0,1,2 .
$$

We set $\tilde{D}:=\sum_{0 \leq i \leq 2} D_{i}$.

We suppose further that the random field is strongly mixing in the uniform sense. More precisely, for any $R>0$ we let $\mathcal{C}_{R}^{i}$ and $\mathcal{C}_{R}^{e}$ be the $\sigma$-algebras generated by the random variables $H(\mathbf{x})$ with $\mathbf{x} \in \mathbb{D}_{R}$ and $\mathbf{x} \in \mathbb{D}_{R}^{c}$ respectively. The uniform mixing coefficient between the $\sigma$-algebras is

$$
\phi(\rho):=\sup \left[|\mathbb{P}(B)-\mathbb{P}(B \mid A)|: R>0, A \in \mathcal{C}_{R}^{i}, B \in \mathcal{C}_{R+\rho}^{e}\right],
$$

for all $\rho>0$. We suppose that $\phi(\rho)$ decays faster than any power: for each $p>0$

$$
h_{p}:=\sup _{\rho \geq 0} \rho^{p} \phi(\rho)<+\infty .
$$

The two-point spatial correlation function of the random field $H$ is $R(\mathbf{y}):=\mathbb{E}[H(\mathbf{y}) H(\mathbf{0})]$. Note that (2.3) implies that for each $p>0$

$$
h_{p}(M):=\sum_{i=0}^{4} \sum_{|\alpha|=i} \sup _{\mathbf{y} \in \mathbb{R}^{2}}\left(1+|\mathbf{y}|^{2}\right)^{p / 2}\left|\partial_{\mathbf{y}}^{\alpha} R(\mathbf{y})\right|<+\infty .
$$

This in turn allows us to define

$$
\hat{R}(\mathbf{v}):=\int R(\mathbf{x}) \exp (-i \mathbf{v} \cdot \mathbf{x}) d \mathbf{x}
$$

the power spectrum of $H$. We assume that the following non-degeneracy condition holds:

(ND) the correlation function $R(\cdot)$ belongs to $C^{\infty}\left(\mathbb{R}^{2}\right)$ and $\hat{R}(\cdot)$ does not vanish identically on any line $H_{\mathbf{p}}=\left\{\mathbf{v} \in \mathbb{R}^{2}: \mathbf{v} \cdot \mathbf{p}=0\right\}$.

\subsection{The main result}

Consider the motion of a particle governed by a Hamiltonian system of equations

$$
\left\{\begin{array}{l}
\dot{\mathbf{z}}^{(\delta)}(t)=\mathbf{w}^{(\delta)}(t) \\
\dot{\mathbf{w}}^{(\delta)}(t)=-\frac{1}{\sqrt{\delta}} \nabla_{\mathbf{z}} H\left(\frac{\mathbf{z}^{(\delta)}(t)}{\delta}\right) \\
\mathbf{z}^{(\delta)}(0)=\mathbf{x}, \quad \mathbf{w}^{(\delta)}(0)=\mathbf{v},
\end{array}\right.
$$

where the potential $H(\mathbf{x})$ is a random field satisfying assumptions in Section 2.2. This motion preserves the total Hamiltonian: $\mathcal{H}^{\delta}(\mathbf{x}, \mathbf{v})=|\mathbf{v}|^{2} / 2+\sqrt{\delta} H(\mathbf{x})$, i.e.

$$
\mathcal{H}^{\delta}\left(\mathbf{z}^{(\delta)}(t), \mathbf{w}^{(\delta)}(t)\right):=\frac{\left|\mathbf{w}^{(\delta)}(t)\right|^{2}}{2}+\sqrt{\delta} H\left(\frac{\mathbf{z}^{(\delta)}(t)}{\delta}\right) \equiv \text { const, } \quad \forall t \geq 0 .
$$


Therefore, as $H(\mathbf{x})$ is uniformly bounded by a deterministic constant $D_{0}$ (see (2.2) $),\left|\mathbf{w}^{(\delta)}(t)\right|$ stays uniformly close to $\left|\mathbf{w}^{(\delta)}(0)\right|=|\mathbf{v}|$ for all $t \geq 0$. In order to formalize this, for a given $M>10$ and $\delta_{*}>0$ sufficiently small (depending on $M$ and $\tilde{D}$ ) we let

$$
\left.M_{*}:=\max \left[\left(M^{2} / 2+2 \delta_{*}^{1 / 2} \tilde{D}\right)^{1 / 2}\right)^{1 / 2},\left[1 /\left(2 M^{2}\right)-2 \delta_{*}^{1 / 2} \tilde{D}\right]^{-1 / 2}\right] .
$$

Then, (2.6) implies that for a particle that is governed by the Hamiltonian flow generated by (2.5) we have

$$
M_{*}^{-1} \leq\left|\mathbf{w}^{(\delta)}(t)\right| \leq M_{*}
$$

for all $t$ provided that the initial data $(\mathbf{x}, \mathbf{v})) \in \mathcal{A}(M)$ and $0<\delta<\delta_{*}$. Accordingly, we define $\mathcal{C}\left(M_{*}\right)$ as the subspace of $\mathcal{C}$ containing paths $\pi=(X(\cdot), V(\cdot))$ for which $\left(2 M_{*}\right)^{-1} \leq|V(t)| \leq 2 M_{*}$, $X(t)$ is differentiable in time, and $\dot{X}(t)=V(t)$ for all $t \geq 0$. The inequality (2.7) means that the trajectory $\left(\mathbf{z}^{(\delta)}(t ; \mathbf{x}, \mathbf{v}), \mathbf{w}^{(\delta)}(t ; \mathbf{x}, \mathbf{v})\right), \delta \in\left(0, \delta_{*}\right]$ necessarily lies in $\mathcal{C}\left(M_{*}\right)$, provided that the initial data $(\mathbf{x}, \mathbf{v}) \in \mathcal{A}(M)$. We denote by $Q_{s, \mathbf{x}, \mathbf{v}}^{\delta}(\cdot)$ the law over $\mathcal{C}$ of the process corresponding to (2.5) starting at $t=s$ from $(\mathbf{x}, \mathbf{v})$ (this law is actually supported in $\mathcal{C}\left(M_{*}\right)$ ). We shall omit writing the subscript $s$ when it equals to 0 .

We now describe the limit process as $\delta \downarrow 0$. Let $(\mathbf{v}(t))_{t \geq 0}$ be a diffusion, starting at $\mathbf{v} \in \mathbb{R}_{*}^{2}$ at $t=0$, with the generator of the form

$$
\mathcal{L} F(\mathbf{v})=\sum_{m, n=1}^{2} D_{m n}(\mathbf{v}) \partial_{v_{m}, v_{n}}^{2} F(\mathbf{v})+\sum_{m=1}^{2} E_{m}(\mathbf{v}) \partial_{v_{m}} F(\mathbf{v})=\sum_{m, n=1}^{2} \partial_{v_{m}}\left(D_{m, n}(\mathbf{v}) \partial_{v_{n}} F(\mathbf{v})\right),
$$

defined for $F \in C_{0}^{\infty}\left(\mathbb{R}_{*}^{2}\right)$. Here the diffusion matrix is given by

$$
D_{m n}(\mathbf{v})=-\frac{1}{2 v} \int_{-\infty}^{+\infty} \partial_{x_{n}, x_{m}}^{2} R(s \hat{\mathbf{v}}) d s, \quad m, n=1,2
$$

and the drift vector is

$$
E_{m}(\mathbf{v})=-\frac{1}{v^{2}} \sum_{n=1}^{2} \int_{0}^{+\infty} s \Delta R_{m}(s \hat{\mathbf{v}}) d s, \quad m=1,2,
$$

where $\Delta:=\partial_{y_{1}}^{2}+\partial_{y_{2}}^{2}$ stands for the two dimensional Laplacian and $R_{j}(\cdot):=\partial_{y_{j}} R(\cdot)$. Employing exactly the same argument as the one used in Section 4 of [1] it can be easily seen that $\mathbf{v}(t)$ is a degenerate diffusion in $\mathbb{R}_{*}^{2}$ supported on the circle $\mathbb{S}_{v}$. Moreover, it is a non-degenerate diffusion on the circle under the non-degeneracy hypotheses (ND) made about $\hat{R}(\cdot)$, cf. Proposition 4.3 of ibid. Suppose that $\mathbf{v}(0)=\mathbf{v} \neq \mathbf{0}$ and denote by $\mathfrak{Q}_{\mathbf{x}, \mathbf{v}}$ the law, over $\mathcal{C}$, of the process $\left(\mathbf{x}+\int_{0}^{t} \mathbf{v}(s) d s, \mathbf{v}(t)\right)$. The joint process is a degenerate diffusion, whose generator equals

$$
\tilde{\mathcal{L}} F(\mathbf{x}, \mathbf{v})=\mathcal{L}_{\mathbf{v}} F(\mathbf{x}, \mathbf{v})+\mathbf{v} \cdot \nabla_{\mathbf{x}} F(\mathbf{x}, \mathbf{v}), \quad F \in C_{0}^{\infty}\left(\mathbb{R}^{2} \times \mathbb{S}_{v}\right) .
$$

Here the notation $\mathcal{L}_{\mathbf{v}}$ stresses that the operator $\mathcal{L}$ defined in (2.8) acts on the respective function in the $\mathbf{v}$ variable. We denote by $\mathfrak{M}_{\mathbf{x}, \mathbf{v}}$ the expectation corresponding to the path measure $\mathfrak{Q}_{\mathbf{x}, \mathbf{v}}$. The main result of this paper can be stated as follows.

Theorem 2.1 Suppose that the random field $H$ satisfies the assumptions made in Section [2.2. Assume also that $\mathbf{v} \neq \mathbf{0}$. Then, the laws $Q_{\mathbf{x}, \mathbf{v}}^{(\delta)}$ converge weakly, as $\delta \rightarrow 0$, to $\mathfrak{Q}_{\mathbf{x}, \mathbf{v}}$. 
The overall strategy of the proof of Theorem 2.1 is similar to that in [1, 4, 15. Briefly, it can be summarized as follows. The Hamiltonian system (2.5) is modified in such a way that the particle can not "turn back violently". This ensures that the particle "moves forward", at least locally in time. This was done by multiplying the right side of (2.5) by a cut-off function $\Theta(t, X(t), V(t) ; \pi)$ that depends both on the current position $(X(t), V(t))$ and on the past trajectory $\pi$. More precisely, one introduces a time mesh $t_{k}^{(1)}=1 / p_{1}$ - the function $\Theta$ is equal to zero on the time interval $\left[t_{k}^{(1)}, t_{k+1}^{(1)}\right]$ if the momentum $V(t)$ is far from $V\left(t_{k}^{(1)}\right)$. This keeps momenta aligned on the time scale $p_{1}^{-1}$ and propels the particle forward. The second cut-off requires that if $X(t), t \in\left[t_{k}^{(1)}, t_{k+1}^{(1)}\right]$ is close to $X(s)$ with $s \leq t_{k-1}^{(1)}$ then $\Theta=0$ and dynamics is trivial. The latter assumption ensures that "no information is gained" at self-intersections. Using the improved mixing properties of the modified dynamics it is possible to show that the modified process converges to the Fokker-Planck diffusion with the generator (2.11). The last step is to observe that for a diffusion in dimension $d \geq 3$ the cut-off $\Theta=1$ for a very long time: it does not make a violent turn on a short time interval, nor does it come close to its past trajectory. Therefore, the modified process, being close to diffusion, also does neither of those things. Hence the stopping times, associated with the first violent turn and coming back, tend to infinity for the modified process. However, until the stopping time the laws of the true process and of its modification, coincide, therefore the stopping time for the original process also tends to infinity, and we conclude that the law of the momentum of the true solution of the Hamiltonian system also approaches the law of the Brownian motion on a sphere as $\delta \rightarrow 0$.

As we have mentioned in the introduction, the two-dimensional case requires a special treatment because the limit diffusion intersects its past - hence the idea of "always seeing a new randomness" may not work. However, one can show that the limit process does not intersect the past trajectory nearly tangentially. Therefore, the time it stays close to its past is small - this provides the key hope for the proof. Accordingly, we modify the Kesten-Papanicolaou self-intersection cut-off: the particle is forced to go along the straight line ("no information gain") only if it intersects the past trajectory non-transversally. Transversal intersections undergo the original dynamics. The non-transversality condition of self-intersections is formalized as follows. First, we set-up the mesh $t_{k}^{(1)}$ on which we require the momentum alignment, as in [1, 4, 5]. In addition, we set-up a finer mesh $t_{k}^{(2)}=k / p_{2}$ with $p_{2} \gg p_{1}$ and impose that $\Theta(t)=0$ if at time $t$ the position $X(t)$ is close to $X\left(t_{k}^{(2)}\right)$ with $t_{k}^{(2)}<t-p_{1}^{-1}$ and the directions $\hat{V}(t)$ and $\hat{V}\left(t_{k}^{(2)}\right)$ are nearly aligned. Since non-transversality is checked only for times on the $1 / p_{2}$-mesh, we have to introduce an additional cut-off that requires the momenta to be aligned on this time scale. This means that the momenta at self-intersections are transversal to those at all times on the interval $t \in\left[t_{k}^{(2)}, t_{k+1}^{p(2)}\right]$, and not only the discrete times on the mesh. That allows one to bound the total time spent near the past trajectory in the modified dynamics: see Proposition 3.3 This bound allows us to bound the "correlation gain" at self-intersections and proceed to the next step - we establish an approximate martingale equality in Proposition 3.5 for the modified dynamics. However, this property holds only with some time delay: $u-t \geq p_{3}^{-1}$ with an appropriate $p_{3}$ - this forces us to introduce a third time mesh $t_{k}^{(3)}=k / p_{3}$ as well as cut-offs that prohibit a violent turn on this time scale. This is required to control the times when the approximate martingale equality fails.

The rest of the proof is similar to [5]: we consider a concatenated process. It follows the modified dynamics until the stopping time, when one of the aforementioned events occur, and the limit diffusion after this time. We use the approximate martingale property of the modified process to show that the concatenated process converges to the correct diffusive limit. That means that the stopping time for the concatenated process has to go to infinity as $\delta \downarrow 0$. However, until the stopping time the concatenated, modified and original processes all coincide, hence the modified process also 
has the right limit, as well as the original dynamics, and the proof is complete.

\section{The cut-off dynamics}

In this section we introduce the modified dynamics and establish the tightness of the family of processes with the cut-offs.

\subsection{The stopping times}

First, we explain the stopping times that we will need in the proof of Theorem 2.1 Let $\epsilon_{i}, i=1, \ldots, 8$ be certain positive constants. We set

$$
\begin{aligned}
& p_{1}=\left[\delta^{-\epsilon_{1}}\right], \quad p_{2}=p_{1}\left[\delta^{-\epsilon_{2}}\right], \quad p_{3}:=p_{1}\left[\delta^{-\epsilon_{3}}\right], \quad p_{4}:=\left[\delta^{-\epsilon_{4}}\right], \\
& N_{1}=\left[\delta^{-\epsilon_{5}}\right], \quad N_{2}:=N_{4}\left[\delta^{-\epsilon_{6}}\right], \quad N_{3}:=\left[\delta^{-\epsilon_{7}}\right], \quad N_{4}:=\left[\delta^{-\epsilon_{8}}\right] .
\end{aligned}
$$

We will specify the restrictions on the constants $\epsilon_{i}$ as the need for such constraints arises. However, the basic requirement is that they should be sufficiently small. At this time we assume only that all the parameters appearing in (3.1) are less than $\delta^{-1}$. This forces upon us the assumptions

$$
\epsilon_{1}, \epsilon_{4}, \epsilon_{5}, \epsilon_{7}, \epsilon_{8} \in(0,1), \quad \epsilon_{1}+\epsilon_{2}, \epsilon_{1}+\epsilon_{3}, \epsilon_{6}+\epsilon_{8} \in(0,1) .
$$

We introduce the following $\left(\mathcal{M}^{t}\right)_{t \geq 0}$-stopping times. Consider a path $\pi \in \mathcal{C}\left(M_{*}\right)$ and let $t_{k}^{(i)}:=k p_{i}^{-1}$, $i=1,2,3$. We define the three "violent turn" stopping times on each scale corresponding to the mesh sizes $p_{i}^{-1}, i=1,2,3$ :

$$
\begin{aligned}
& S_{\delta}^{(i)}(\pi):=\inf \left[t \geq 0: \text { for some } k \geq 0 \text { we have } t \in\left[t_{k}^{(i)}, t_{k+1}^{(i)}\right)\right. \text { s.t. } \\
& \left.\hat{V}\left(t_{k-1}^{(i)}\right) \cdot \hat{V}(t) \leq 1-\frac{1}{N_{i}}, \text { or }\left|V\left(t_{k}^{(i)}\right)-V(t)\right| \geq \frac{1}{\left(2 N_{i}\right)^{1 / 2} M_{*}}\right] .
\end{aligned}
$$

This ensures that during a time interval of length $1 / p_{i}$ the particle momentum does not change by more than $O\left(N_{i}^{-1 / 2}\right)$ - the particle goes forward. We shall assume that $N_{i} \ll p_{i}, i=1,2,3$, which holds if

$$
\epsilon_{5}<\epsilon_{1}, \quad \epsilon_{6}+\epsilon_{8}<\epsilon_{1}+\epsilon_{2}, \quad \epsilon_{7}<\epsilon_{1}+\epsilon_{3} .
$$

This condition guarantees that the expected limit diffusion process should have the same property. Note that for any path $\pi \in \mathcal{C}\left(M_{*}\right)$ the condition

$$
\left|V\left(t_{k}^{(i)}\right)-V(t)\right| \leq M_{*}^{-1}\left(2 N_{i}\right)^{-1 / 2}
$$

implies $\hat{V}\left(t_{k}^{(i)}\right) \cdot \hat{V}(t) \geq 1-1 / N$ - hence until the stopping time the momenta directions are aligned on the corresponding time scales. This fact follows from an elementary inequality $|\hat{x}-\hat{y}| \leq 2 M_{*}|x-y|$ for arbitrary $x, y \in \mathbb{R}^{d}$ satisfying $|x|,|y| \geq\left(2 M_{*}\right)^{-1}$. We note that both in the definition of the stopping times above and elsewhere we adopt the convention that the infimum of an empty set equals $+\infty$.

The last stopping time deals with the path self-intersections. For each $t \geq 0$, we denote by

$$
\mathfrak{X}_{t}(\pi):=\bigcup_{0 \leq s \leq t} X(s ; \pi)
$$


the trace of the spatial component of the path $\pi$ up to time $t$, and by

$$
\mathfrak{X}_{t}\left(p_{2} ; \pi\right):=\left[\mathbf{x}: \operatorname{dist}\left(\mathbf{x}, \mathfrak{X}_{t}(\pi)\right) \leq 1 / p_{2}\right]
$$

a tubular region ("sausage") around the path. We introduce the following stopping time

$$
\begin{aligned}
& U_{\delta}(\pi):=\inf \left[t \geq 0: \exists k \geq 1, s \in\left[0, t_{k-1}^{(1)}\right], t \in\left[t_{k}^{(1)}, t_{k+1}^{(1)}\right), \text { for which }|X(t)-X(s)|<\frac{1}{p_{2}}\right. \\
& \left.\quad \text { and }|\hat{V}(t) \cdot \hat{V}(s)| \geq 1-\frac{1}{N_{4}}\right] .
\end{aligned}
$$

Any path self-intersections are transversal until the time $U_{\delta}$. Moreover, since $N_{2} \gg N_{4}$, the intersection angle is much larger than the oscillations of the trajectory on the $1 / p_{2}$-time scale. This condition will be used below to control the time that a piece of the future may spend in the sausage of width $1 / p_{4}$ around the past. This is the role of the parameter $p_{4}$. It will be made precise later on: see (3.23) and Proposition 3.3

Finally, we set the stopping times

$$
S_{\delta}(\pi):=S_{\delta}^{(1)}(\pi) \wedge S_{\delta}^{(2)}(\pi) \wedge S_{\delta}^{(3)}(\pi)
$$

and

$$
\tau_{\delta}(\pi):=S_{\delta}(\pi) \wedge U_{\delta}(\pi)
$$

\subsection{The cut-off functions}

We define first the cut-off functions needed for the "no violent turn" stopping times. Let $M>10$ be fixed and suppose that the function $\psi_{1}: \mathbb{R}^{2} \times \mathbb{S} \times \mathbb{Z}_{+} \rightarrow[0,1]$ is of the $C^{\infty}$ class and such that

$$
\psi_{1}(\mathbf{v}, \mathbf{l}, K)=\left\{\begin{array}{lll}
1, & \text { if } \quad \hat{\mathbf{v}} \cdot \mathbf{l} \geq 1-1 / K & \text { and } \quad M_{*}^{-1} \leq|\mathbf{v}| \leq M_{*} \\
0, & \text { if } \quad \hat{\mathbf{v}} \cdot \mathbf{l} \leq 1-2 / K, & \text { or } \quad|\mathbf{v}| \leq\left(2 M_{*}\right)^{-1}, \quad \text { or } \quad|\mathbf{v}| \geq 2 M_{*} .
\end{array}\right.
$$

Let also $\psi_{2}: \mathbb{R}_{*}^{2} \times \mathbb{R}_{*}^{2} \times \mathbb{Z}_{+} \rightarrow[0,1]$ be a $C^{\infty}$ class function that in addition satisfies

$$
\psi_{2}(\mathbf{v}, \mathbf{l}, K)= \begin{cases}1, & \text { if } \quad|\mathbf{v}-\mathbf{l}| \leq M_{*}^{-1}(2 K)^{-1 / 2} \\ 0, & \text { if } \quad|\mathbf{v}-\mathbf{l}| \geq M_{*}^{-1} K^{-1 / 2}\end{cases}
$$

One can construct $\psi$ in such a way that for arbitrary nonnegative integers $m, n$ there exists a constant $C_{m, n}$ so that $\left\|\psi_{j}\right\|_{m, n} \leq C_{m, n} K^{(m+n) / 2}, j=1,2$. Suppose now that the reciprocal mesh sizes $p_{i}$ and "angle turn cut-off" amplitudes $N_{i}$ are the positive integers defined in Section 3.1. Define the $1 / p_{i}$-scale "violent turn" cut-off function as

$$
\Psi_{i}(t, \mathbf{v} ; \pi):= \begin{cases}\psi_{1}\left(\mathbf{v}, \hat{V}\left(t_{k-1}^{(i)}\right), N_{i}\right) \psi_{2}\left(\mathbf{v}, V\left(t_{k}^{(i)}\right), N_{i}\right) & \text { for } t \in\left[t_{k}^{(i)}, t_{k+1}^{(i)}\right) \text { and } k \geq 1, \\ \psi_{2}\left(\mathbf{v}, V(0), N_{i}\right) & \text { for } t \in\left[0, t_{1}^{(i)}\right) .\end{cases}
$$

The next step is to introduce the cut-off that prevents nearly tangential self-intersections. It is defined as follows. Let the function $\phi: \mathbb{R}_{*}^{4} \times \mathbb{R}_{*}^{4} \rightarrow[0,1]$ be of the $C^{\infty}$ class and satisfy $\phi(\mathbf{y}, \mathbf{v} ; \mathbf{x}, \mathbf{w})=$ 1 , when $|\mathbf{y}-\mathbf{x}| \geq 1 / p_{2}$, or $|\hat{\mathbf{v}} \cdot \hat{\mathbf{w}}| \leq 1-1 / N_{4}$ and $\phi(\mathbf{y}, \mathbf{v} ; \mathbf{x}, \mathbf{w})=0$, when both $|\mathbf{y}-\mathbf{x}| \leq 1 /\left(2 p_{2}\right)$ and $|\hat{\mathbf{v}} \cdot \hat{\mathbf{w}}| \geq 1-1 /\left(2 N_{4}\right)$. Again, in this case we can construct $\phi$ in such a way that $\|\phi\|_{m_{1}, m_{2}, n_{1}, n_{2}} \leq$ 
$C_{m n} p_{2}^{m_{1}+n_{1}} N_{4}^{m_{2}+n_{2}}$ for arbitrary integers $m_{1}, m_{2}, n_{1}, n_{2}$ and a suitably chosen constant $C_{m n}$. The cut-off function $\phi_{k}: \mathbb{R}_{*}^{4} \times \mathcal{C} \rightarrow[0,1]$ for a fixed path $\pi$ is given by

$$
\phi_{k}(\mathbf{y}, \mathbf{v} ; \pi)=\prod_{0 \leq t_{l}^{(2)} \leq t_{k-1}^{(1)}} \phi\left(\mathbf{y}, \mathbf{v} ; X\left(t_{l}^{(2)}\right), V\left(t_{l}^{(2)}\right)\right) .
$$

We set

$$
\Phi(t, \mathbf{y}, \mathbf{v} ; \pi):= \begin{cases}1, & \text { if } 0 \leq t<t_{1}^{(1)} \\ \phi_{k}(\mathbf{y}, \mathbf{v} ; \pi), & \text { if } t_{k}^{(1)} \leq t<t_{k+1}^{(1)} .\end{cases}
$$

For a given $t \geq 0,(\mathbf{y}, \mathbf{v}) \in \mathbb{R}_{*}^{4}$ and a path $\pi \in \mathcal{C}$ let us define the cut-off function

$$
\Theta(t, \mathbf{y}, \mathbf{v} ; \pi):=\Phi(t, \mathbf{y}, \mathbf{v} ; \pi) \prod_{i=1}^{3} \Psi_{i}(t, \mathbf{v} ; \pi)
$$

that incorporates all of the necessary cut-offs. In the sequel we will need the result of the following lemma that can be verified by a direct calculation.

Lemma 3.1 Let $\left(\beta_{1}, \beta_{2}\right)$ be a multi-index with nonnegative integer valued components, $m=\left|\beta_{1}\right|+$ $\left|\beta_{2}\right|$ and $T>0$. Then there exists a constant $C$ depending only on $m$ and $M$ such that

$$
\left|\partial_{\mathbf{y}}^{\beta_{1}} \partial_{\mathbf{v}}^{\beta_{2}} \Theta(t, \mathbf{y}, \mathbf{v} ; \pi)\right| \leq C p_{2}^{2\left|\beta_{1}\right|+\left|\beta_{2}\right|}\left(N_{1} N_{2} N_{3} N_{4}\right)^{\left|\beta_{2}\right| / 2}
$$

for all $t \in[0, T],(\mathbf{y}, \mathbf{v}) \in \mathcal{A}(2 M), \pi \in \mathcal{C}\left(M_{*}\right)$.

We note that the power of $p_{2}$ appears due to two contributions: one comes from the dependence of $\phi$ on $\mathbf{y}$, and another from the number of terms in the product in the definition (3.11) of the function $\phi_{k}$ that arise both when differentiating the function $\Theta$ in $\mathbf{y}$ and in $\mathbf{v}$. We also define the re-scaled function

$$
\Theta_{\delta}(t, \mathbf{y}, \mathbf{l} ; \pi):=\Theta(t, \delta \mathbf{y}, \mathbf{l} ; \pi) .
$$

Observe that according to Lemma 3.1 we have

$$
\left|\partial_{\mathbf{y}}^{\beta_{1}} \partial_{\mathbf{v}}^{\beta_{2}} \Theta_{\delta}(t, \mathbf{y}, \mathbf{l})\right| \leq C \delta^{\left|\beta_{1}\right|\left[1-2\left(\epsilon_{1}+\epsilon_{2}\right)\right]}\left(p_{2} N_{1} N_{2} N_{3} N_{4}\right)^{\left|\beta_{2}\right| / 2} \leq C\left(p_{2} N_{1} N_{2} N_{3} N_{4}\right)^{\left|\beta_{2}\right| / 2},
$$

provided that

$$
2\left(\epsilon_{1}+\epsilon_{2}\right)<1
$$

\subsection{The modified dynamics in two dimensions}

We are now ready to define the modified dynamics with the cut-offs and describe some of its elementary geometric properties in two dimensions. Given a path $\pi \in \mathcal{C}$ we introduce the vector field

$$
F_{\delta}(t, \mathbf{y}, \mathbf{l} ; \pi, \omega)=\Theta_{\delta}(t, \mathbf{y}, \mathbf{l} ; \pi) \nabla_{\mathbf{y}} H(\mathbf{y} ; \omega),
$$

with the cut-off function $\Theta_{\delta}$ defined in (3.13).

For a fixed $(\mathbf{x}, \mathbf{v}) \in \mathbb{R}_{*}^{4}, \delta>0$ and realization $\omega \in \Omega$ we consider the modified particle dynamics with the cut-off that is described by the stochastic process $\left(\boldsymbol{y}^{(\delta)}(t ; \mathbf{x}, \mathbf{v}, \omega), \boldsymbol{l}^{(\delta)}(t ; \mathbf{x}, \mathbf{v}, \omega)\right)$ whose paths are the solutions of the following equation

$$
\left\{\begin{array}{l}
\dot{y}^{(\delta)}(t ; \mathbf{x}, \mathbf{v})=\boldsymbol{l}^{(\delta)}(t ; \mathbf{x}, \mathbf{k}) \\
\dot{\boldsymbol{i}}^{(\delta)}(t ; \mathbf{x}, \mathbf{v})=-\frac{1}{\sqrt{\delta}} F_{\delta}\left(t, \frac{\boldsymbol{y}^{(\delta)}(t ; \mathbf{x}, \mathbf{v})}{\delta}, \boldsymbol{l}^{(\delta)}(t ; \mathbf{x}, \mathbf{v}) ; \boldsymbol{y}^{(\delta)}(\cdot ; \mathbf{x}, \mathbf{v}), \boldsymbol{l}^{(\delta)}(\cdot ; \mathbf{x}, \mathbf{v})\right) \\
\boldsymbol{y}^{(\delta)}(0 ; \mathbf{x}, \mathbf{v})=\mathbf{x}, \quad \boldsymbol{l}^{(\delta)}(0 ; \mathbf{x}, \mathbf{v})=\mathbf{v}
\end{array}\right.
$$


We will denote by $\tilde{Q}_{\mathbf{x}, \mathbf{v}}^{(\delta)}$ the law of $\left(\boldsymbol{y}^{(\delta)}(\cdot ; \mathbf{x}, \mathbf{v}), \boldsymbol{l}^{(\delta)}(\cdot ; \mathbf{x}, \mathbf{v})\right)$ over $\mathcal{C}$ and by $\tilde{E}_{\mathbf{x}, \mathbf{v}}^{(\delta)}$ the corresponding expectation. ¿From the construction of the cut-offs we deduce the following geometric properties of the trajectories $\left(\boldsymbol{y}^{(\delta)}(\cdot), \boldsymbol{l}^{(\delta)}(\cdot)\right)$.

Lemma 3.2 Suppose that $(\mathbf{x}, \mathbf{v}) \in \mathcal{A}(M)$. If for some $s_{0} \in \bigcap_{i=1}^{3}\left[t_{j_{i}}^{(i)}, t_{j_{i}+1}^{(i)}\right)$ and $t_{0} \in \bigcap_{i=1}^{3}\left[t_{k_{i}}^{(i)}, t_{k_{i}+1}^{(i)}\right)$, where $j_{1}<k_{1}$ we have $\Theta\left(t_{0}, \delta \boldsymbol{y}^{(\delta)}\left(t_{0}\right), \boldsymbol{l}^{(\delta)}\left(t_{0}\right) ; \boldsymbol{y}^{(\delta)}, \boldsymbol{l}^{(\delta)}\right) \neq 0$ and $\left|\boldsymbol{y}^{(\delta)}\left(t_{0}\right)-\boldsymbol{y}^{(\delta)}\left(s_{0}\right)\right| \leq 1 /\left(2 p_{2}\right)$ then

$$
\left|\hat{\boldsymbol{l}}^{(\delta)}(t) \cdot \hat{\boldsymbol{l}}^{(\delta)}(s)\right| \leq 1-\frac{1}{4 N_{4}}, \quad \forall s \in\left[t_{j_{2}}^{(2)}, t_{j_{2}+1}^{(2)}\right), \quad t \in\left[t_{k_{2}}^{(2)}, t_{k_{2}+1}^{(2)}\right)
$$

provided that $\delta \in\left(0, \delta_{*}\right]$ and $\delta_{*}>0$ is sufficiently small. Moreover, for all $k \geq 1, i=1,2,3$ we have

$$
\begin{aligned}
& \hat{\boldsymbol{l}}^{(\delta)}(t) \cdot \hat{\boldsymbol{l}}^{(\delta)}\left(t_{k-1}^{(i)}\right) \geq 1-\frac{2}{N_{i}}, \quad \forall t \in\left[t_{k-1}^{(i)}, t_{k+1}^{(i)}\right), \\
& \hat{\boldsymbol{l}}^{(\delta)}(t) \cdot \hat{\boldsymbol{l}}^{(\delta)}\left(t_{k-1}^{(i)}\right) \geq 1-\frac{18}{N_{i}}, \quad \forall t \in\left[t_{k+1}^{(i)}, t_{k+2}^{(i)}\right),
\end{aligned}
$$

and

$$
\hat{\boldsymbol{l}}^{(\delta)}(t) \cdot \hat{\boldsymbol{l}}^{(\delta)}(s) \geq 1-\frac{8}{N_{i}}, \quad \forall t, s \in\left[t_{k-1}^{(i)}, t_{k}^{(i)}\right),
$$

provided that $\delta \in\left(0, \delta_{*}\right]$ and $\delta_{*}>0$ is sufficiently small.

The proof of this Lemma is elementary and is contained in Appendix A. We show next that a consequence of Lemma 3.2 is that the modified trajectory stays only for a little time in a tube around its past as long as the cut-offs are not equal to zero. We assume that

$$
\epsilon_{4} \in(1 / 2,1)
$$

that is, $p_{4}$ is larger than all other cut-off parameters defined in (3.1). For any $k \in \mathbb{Z}$ we define

$$
\mathfrak{X}_{\delta}\left(k, p_{4}\right):= \begin{cases}\emptyset, & \text { if } k<0, \\ \mathfrak{X}_{t_{k}^{(1)}}\left(p_{4} ; \boldsymbol{y}^{(\delta)}(\cdot), \boldsymbol{l}^{(\delta)}(\cdot)\right), & \text { if } k \geq 0,\end{cases}
$$

the tube of the size $1 / p_{4}$ around the trajectory until the time $t_{k}^{(1)}$. Let also

$$
B_{\delta}\left(k, p_{4} ; \omega\right):=\left[t \in\left[t_{k}^{(1)}, t_{k+2}^{(1)}\right]: \boldsymbol{y}^{(\delta)}(t) \in \mathfrak{X}_{\delta}\left(k-1, p_{4}\right) \text { and } \Theta_{\delta}\left(t, \boldsymbol{y}^{(\delta)}(t), \boldsymbol{l}^{(\delta)}(t) ; \boldsymbol{y}^{(\delta)}(\cdot), \boldsymbol{l}^{(\delta)}(\cdot)\right) \neq 0\right]
$$

be the set of times spent by the trajectory during the time interval $\left[t_{k}^{(1)}, t_{k+2}^{(1)}\right]$ in a narrow tube around the past and in a direction transversal to the tube. The following proposition gives an upper bound on the measure of this set.

Proposition 3.3 Suppose that $(\mathbf{x}, \mathbf{v}) \in \mathcal{A}(M)$ and $k \geq 1$. Then, there exists a deterministic constant $C>0$ such that

$$
m_{1}\left[B_{\delta}\left(k, p_{4} ; \omega\right)\right] \leq C \frac{N_{4}^{1 / 2} p_{2}^{2}}{p_{1} p_{4}}, \quad \mathbb{P}-\text { a.s. },
$$

provided that $\delta \in\left(0, \delta_{*}\right]$, where $\delta_{*}>0$ is sufficiently small. Here $m_{1}$ is the one-dimensional Lebesgue measure. 
Proof. For any integer $l \geq 0$ we denote by $\Gamma_{l}$ the $\operatorname{arc} \boldsymbol{y}^{(\delta)}(s), s \in\left[t_{l}^{(2)}, t_{l+1}^{(2)}\right]$ and by

$$
G_{l}:=\left[\mathbf{y} \in \mathbb{R}^{2}: \operatorname{dist}\left(\mathbf{y}, \Gamma_{l}\right) \leq 1 / p_{4}\right]
$$

the $1 / p_{4}$-neighborhood of $\Gamma_{l}$. We fix one interval $\left[t_{i}^{(2)}, t_{i+1}^{(2)}\right] \subseteq\left[t_{k}^{(1)}, t_{k+2}^{(1)}\right]$ and assume that there exists

$$
t \in B_{\delta}^{i}\left(k, p_{4} ; \omega\right):=B_{\delta}\left(k, p_{4} ; \omega\right) \cap\left[t_{i}^{(2)}, t_{i+1}^{(2)}\right] .
$$

This means that there exists a sub-interval $\left[t_{j}^{(2)}, t_{j+1}^{(2)}\right] \subseteq\left[0, t_{k-1}^{(1)}\right]$ so that the curve $\Gamma_{i}$ intersects the tube $G_{j}$. Let $\sigma_{1}, \sigma_{2}$ be two subsequent exit and entrance times of $\Gamma_{i}$ into $G_{j}$, that is, we let first $\sigma_{0}:=\min \left[s \in\left[t_{i}^{(2)}, t_{i+1}^{(2)}\right]: \boldsymbol{y}^{(\delta)}(s) \in G_{j}\right]$ and then

$$
\begin{gathered}
\sigma_{1}:=\inf \left[s \in\left[t_{i}^{(2)}, t_{i+1}^{(2)}\right]: s \geq \sigma_{0}, \boldsymbol{y}^{(\delta)}(s) \in G_{j}^{c}\right], \\
\sigma_{2}:=\min \left[s \in\left[t_{i}^{(2)}, t_{i+1}^{(2)}\right]: s>\sigma_{1}, \boldsymbol{y}^{(\delta)}(s) \in G_{j}\right] .
\end{gathered}
$$

We recall here our convention that the stopping times equals $+\infty$ if the respective sets are empty.

As a consequence of the transversality condition (3.18) and the slow variation of the tangent field expressed by (3.21) we conclude, see Lemma B.1 in Appendix, that $\sigma_{2}=+\infty$ - the particle may not re-enter the tube $G_{j}$ during the time interval $\left[t_{i}^{(2)}, t_{i+1}^{(2)}\right]$ if it goes through $G_{j}$ transversally. Thus, the intersection $\Gamma_{i} \cap G_{j}$ is connected and the set

$$
B_{\delta}^{i j}\left(k, p_{4}, \omega\right):=\left[t \in\left[t_{i}^{(2)}, t_{i+1}^{(2)}\right]: \boldsymbol{y}^{(\delta)}(t) \in G_{j}\right]
$$

is actually a time interval. It also follows from (3.18) that the length of the interval $B_{\delta}^{i j}\left(k, p_{4}, \omega\right)$ is at most $C N_{4}^{1 / 2} / p_{4}$ - see Fig. 1 below. Since the whole tube $\mathfrak{X}_{t_{k-1}^{(1)}}\left(p_{4}\right)$ is contained in the union of $G_{j}$, where $0 \leq j \leq([T]+1) p_{2}$ the $m_{1}$ measure of the set

$$
B_{\delta}^{i}\left(k, p_{4}, \omega\right):=\left[t \in\left[t_{i}^{(2)}, t_{i+1}^{(2)}\right]: \boldsymbol{y}^{(\delta)}(t) \in \mathfrak{X}_{t_{k-1}^{(1)}}\left(p_{4}\right)\right]
$$

can be estimated therefore from above by $C N_{4}^{1 / 2} p_{2} / p_{4}$. The same argument can be repeated for each subinterval $\left[t_{i}^{(2)}, t_{i+1}^{(2)}\right]$ of $\left[t_{k}^{(1)}, t_{k+2}^{(1)}\right]$ and, since there are $2 p_{2} / p_{1}$ of such intervals, we obtain (3.24).

The upper bound (3.24) is useful provided that $p_{4}$ is sufficiently large - this is why we take it larger than all other parameters in (3.22).

\subsection{Some consequences of the mixing assumption}

We recall in this section some technical lemmas that translate the mixing properties of the random potential into decorrelation properties of trajectories. Let $\mathcal{F}_{t}$ denote the $\sigma$-algebra generated by $\left(\boldsymbol{y}^{(\delta)}(s), \boldsymbol{l}^{(\delta)}(s)\right), s \leq t$. Here we suppress, for the sake of abbreviation, writing the initial data in the notation of the trajectory. In this section we assume that $X_{1}, X_{2}:\left(\mathbb{R} \times \mathbb{R}^{2} \times \mathbb{R}^{4}\right)^{2} \rightarrow \mathbb{R}$ are certain continuous functions, $Z$ is a random variable and $g_{1}, g_{2}$ are $\mathbb{R}^{2}$-valued random vectors. We suppose further that $Z, g_{1}, g_{2}$, are $\mathcal{F}_{t}$-measurable, while $\tilde{X}_{1}, \tilde{X}_{2}$ are random fields of the form

$$
\tilde{X}_{i}(\mathbf{x})=X_{i}\left(H(\mathbf{x}), \nabla_{\mathbf{x}} H(\mathbf{x}), \nabla_{\mathbf{x}}^{2} H(\mathbf{x})\right) .
$$

We also let

$$
U\left(\theta_{1}, \theta_{2}\right):=\mathbb{E}\left[\tilde{X}_{1}\left(\theta_{1}\right) \tilde{X}_{2}\left(\theta_{2}\right)\right], \quad \theta_{1}, \theta_{2} \in \mathbb{R}^{2} .
$$

The following mixing lemma can be proved in the same way as Lemmas 5.2 and 5.3 of [1]. 




Figure 1

Lemma 3.4 (i) Assume that $r, t \geq 0$ and

$$
\inf _{u \leq t}\left|g_{i}-\frac{\boldsymbol{y}^{(\delta)}(u)}{\delta}\right| \geq \frac{r}{\delta},
$$

$\mathbb{P}-$ a.s. on the set $\{Z \neq 0\}$ for $i=1,2$. Then, we have

$$
\left|\mathbb{E}\left[\tilde{X}_{1}\left(g_{1}\right) \tilde{X}_{2}\left(g_{2}\right) Z\right]-\mathbb{E}\left[U\left(g_{1}, g_{2}\right) Z\right]\right| \leq 2 \phi\left(\frac{r}{2 \delta}\right)\left\|X_{1}\right\|_{L^{\infty}}\left\|X_{2}\right\|_{L^{\infty}}\|Z\|_{L^{1}(\Omega)}
$$

(ii) Let $\mathbb{E} X_{1}(\mathbf{0})=0$. Furthermore, we assume that $g_{2}$ satisfies (3.29),

$$
\inf _{u \leq t}\left|g_{1}-\frac{\boldsymbol{y}^{(\delta)}(u)}{\delta}\right| \geq \frac{r+r_{1}}{\delta}
$$

and $\left|g_{1}-g_{2}\right| \geq r_{1} \delta^{-1}$ for some $r_{1} \geq 0, \mathbb{P}$-a.s. on the event $\{Z \neq 0\}$. Then, we have

$$
\left|\mathbb{E}\left[\tilde{X}_{1}\left(g_{1}\right) \tilde{X}_{2}\left(g_{2}\right) Z\right]-\mathbb{E}\left[U\left(g_{1}, g_{2}\right) Z\right]\right| \leq C \phi^{1 / 2}\left(\frac{r}{2 \delta}\right) \phi^{1 / 2}\left(\frac{r_{1}}{2 \delta}\right)\left\|X_{1}\right\|_{L^{\infty}}\left\|X_{2}\right\|_{L^{\infty}}\|Z\|_{L^{1}(\Omega)}
$$

for some absolute constant $C>0$. Here the function $U$ is given by (3.28).

\subsection{Tightness of the cut-off process}

This section contains the proof of tightness for the process with cut-offs. The proof follows in general [1] and [5] with a couple of additional twists. First, one has to use mixing with the additional control of the time spent in the tube around the past from Proposition 3.3. rather than simply discard the times spent near the past as in $d \geq 3$. Second, we obtain a martingale estimate in Proposition 
3.5 below only for slightly separated times $u-t \geq 1 / p_{3}$. Hence, one has to introduce a linear approximation on the time scale $1 / p_{3}$, show that the martingale estimate suffices for the tightness of the linear approximation and deduce tightness for the full process using its uniform closedness to the linear approximation.

Given $(\mathbf{x}, \mathbf{v}) \in \mathbb{R}_{*}^{4}, \pi \in \mathcal{C}$ and $G \in C_{b}^{1,1,3}\left([0,+\infty) \times \mathbb{R}_{*}^{4}\right)$ we introduce

$$
\begin{aligned}
\widehat{\mathcal{L}}_{t} G(t, \mathbf{x}, \mathbf{v} ; \pi): & =\mathbf{v} \cdot \nabla_{\mathbf{x}} G(t, \mathbf{x}, \mathbf{v})+\Theta^{2}(t, X(t), V(t) ; \pi) \mathcal{L}_{\mathbf{v}} G(t, \mathbf{x}, \mathbf{v}) \\
& -\Theta(t, X(t), V(t) ; \pi) \sum_{m, n=1}^{2} \partial_{V_{m}} \Theta(t, X(t), V(t) ; \pi) D_{m, n}(\hat{\mathbf{v}},|\mathbf{v}|) \partial_{v_{n}} G(t, \mathbf{x}, \mathbf{v})
\end{aligned}
$$

and

$$
\widehat{N}_{t}(G):=G(t, X(t), V(t))-G(0, X(0), V(0))-\int_{0}^{t}\left(\partial_{\varrho}+\widehat{\mathcal{L}}_{\varrho}\right) G(\varrho, X(\varrho), V(\varrho) ; \pi) d \varrho .
$$

Throughout this section we shall omit the initial data from the notation for the path.

Proposition 3.5 Assume that $\epsilon_{i} \in\left(0,10^{-3}\right), i \neq 3,4,7$ and $\epsilon_{3} \in(1 / 7,1 / 6), \epsilon_{4} \in(15 / 16,1)$, $\epsilon_{7} \in(1 / 15,1 / 10)$. Suppose that $(\mathbf{x}, \mathbf{v}) \in \mathcal{A}(M)$ and $\zeta \in C_{b}\left(\left(\mathbb{R}_{*}^{4}\right)^{n}\right)$ is nonnegative. Let $0 \leq t_{1}<\ldots<$ $t_{n} \leq t<u \leq T$. Then, there exists a constant $C>0$ such that for any function $G \in C_{b}^{1,1,3}\left([0, T] \times \mathbb{R}_{*}^{4}\right)$ we have

$$
\left|\tilde{E}_{\mathbf{x}, \mathbf{v}}^{(\delta)}\left\{\left[\widehat{N}_{u}(G)-\widehat{N}_{t}(G)\right] \tilde{\zeta}\right\}\right| \leq C \delta^{1 / 6}\left[(u-t) \vee \frac{1}{p_{3}}\right]\|G\|_{1,1,3} \tilde{E}_{\mathbf{x}, \mathbf{v}}^{(\delta)} \tilde{\zeta}
$$

Here $\tilde{\zeta}(\pi):=\zeta\left(X\left(t_{1}\right), V\left(t_{1}\right), \ldots, X\left(t_{n}\right), V\left(t_{n}\right)\right), \pi \in \mathcal{C}$. The choice of the constant $C$ does not depend on $(\mathbf{x}, \mathbf{v}), \delta \in(0,1], \zeta$, times $t_{1}, \ldots, t_{n}, u, t$, or the function $G$.

We recall that $\tilde{E}_{\mathbf{x}, \mathbf{v}}^{(\delta)}$ is the expectation with respect to the cut-off dynamics.

Before proceeding with the proof of the proposition we show first how to conclude from it the tightness of the laws of $\boldsymbol{l}^{(\delta)}(\cdot)$. Define the process $\tilde{\boldsymbol{l}}^{(\delta)}(\cdot)$ by setting $\tilde{\boldsymbol{l}}^{(\delta)}\left(t_{k}^{(3)}\right)=\boldsymbol{l}^{(\delta)}\left(t_{k}^{(3)}\right), k \geq 0$ and then extend its definition via a linear interpolation. Note that thanks to A.2 of Proposition A.1 in Appendix we have

$$
\sup _{t \geq 0}\left|\tilde{\boldsymbol{l}}^{(\delta)}(t)-\boldsymbol{l}^{(\delta)}(t)\right| \leq \frac{C}{N_{3}^{1 / 2}}
$$

The tightness of the family $\boldsymbol{l}^{(\delta)}(\cdot)$ follows from the above estimate and the following proposition.

Proposition 3.6 The laws of the family $\tilde{\boldsymbol{l}}^{(\delta)}(\cdot), \delta \in(0,1]$ are tight over $C\left([0,+\infty), \mathbb{R}^{2}\right)$.

Proof. The argument is analogous to the proof of Theorem 1.4.6 of [8]. We start with the definition of stopping times $\tau_{k}(\pi)$ (the reader should not confuse these stopping times with the stopping times $\tau_{\delta}(\pi)$ defined in (3.7) as they have nothing to do with each other) that determine the $p_{3}$-mesh times at which the $V$ component of the path $\pi$ performs $k$-th oscillation of size $\rho / 8$, where $\rho>0$ is given. Let $\tau_{0}(\pi):=0$ and for any $k \geq 0$ set

$$
\tau_{k+1}(\pi):=\inf \left[t_{j}^{(3)} \geq \tau_{k}(\pi):\left|V\left(t_{j}^{(3)}\right)-V\left(\tau_{k}(\pi)\right)\right| \geq \frac{\rho}{8}\right],
$$

with the convention that $\tau_{n+1}=+\infty$ when $\tau_{n}=+\infty$, or when the respective event is impossible. Let $N_{\#}:=\min \left[n: \tau_{n+1}>T\right]$ and $\delta^{*}:=\min \left[\tau_{n}-\tau_{n-1}: n=1, \ldots, N_{\#}\right]$. Let $h>0$ and $K-$ a positive 
integer - be fixed. Our first task is to estimate the probability $\tilde{Q}_{\mathbf{x}, \mathbf{v}}^{(\delta)}\left[\delta^{*} \leq h\right]$. To that purpose we write

$$
\begin{aligned}
\tilde{Q}_{\mathbf{x}, \mathbf{v}}^{(\delta)}\left[\delta^{*} \leq h\right] \leq & \tilde{Q}_{\mathbf{x}, \mathbf{v}}^{(\delta)}\left[\delta^{*} \leq h, N_{\#} \leq K\right]+\tilde{Q}_{\mathbf{x}, \mathbf{v}}^{(\delta)}\left[N_{\#}>K\right] \\
& \leq \sum_{i=1}^{K} \tilde{Q}_{\mathbf{x}, \mathbf{v}}^{(\delta)}\left[\tau_{i}-\tau_{i-1} \leq h\right]+\tilde{Q}_{\mathbf{x}, \mathbf{v}}^{(\delta)}\left[N_{\#}>K\right]
\end{aligned}
$$

We will estimate the two terms above as follows. First, we have

$$
\tilde{Q}_{\mathbf{x}, \mathbf{v}}^{(\delta)}\left[\tau_{n+1}-\tau_{n} \leq h \mid \mathcal{M}^{\tau_{n}}\right] \leq A_{\rho} h, \quad \forall h>0
$$

with a constant $A_{\rho}$ depending on $\rho$ but not $h$. Second, we will show that there exists $\gamma<1$ such that

$$
\tilde{Q}_{\mathbf{x}, \mathbf{v}}^{(\delta)}\left[N_{\#}>K\right] \leq e^{T} \gamma^{K} .
$$

¿From (3.34), (3.35) and (3.36) we obtain that

$$
\tilde{Q}_{\mathbf{x}, \mathbf{v}}^{(\delta)}\left[\delta^{*} \leq h\right] \leq K A_{\rho} h+e^{T} \gamma^{K} .
$$

Estimate (3.37), see also Lemma 1.4.1 p. 39 of [8], implies that for any $K \in \mathbb{N}$

$$
\mathbb{P}\left[\max \left[\left|\tilde{\boldsymbol{l}}^{(\delta)}(t)-\tilde{\boldsymbol{l}}^{(\delta)}(s)\right|,|t-s| \leq h, t, s \in[0, T]\right] \geq \frac{\rho}{4}\right] \leq K A_{\rho} h+e^{T} \gamma^{K} .
$$

Choosing $K$ large first and then $h$ small this proves that for any $\sigma, \rho>0$ one can find $h>0$ such that

$$
\mathbb{P}\left[\max \left[\left|\tilde{\boldsymbol{l}}^{(\delta)}(t)-\tilde{\boldsymbol{l}}^{(\delta)}(s)\right|,|t-s| \leq h, t, s \in[0, T]\right] \geq \frac{\rho}{4}\right] \leq \sigma, \quad \forall \delta \in(0,1],
$$

hence the family of laws of $\tilde{\boldsymbol{l}}^{(\delta)}(\cdot)$ is tight on $C\left([0, T] ; \mathbb{R}^{2}\right)$ for all $T>0$ and the conclusion of Proposition 3.6 follows.

It remains to prove (3.35) and (3.36). Let $f: \mathbb{R}^{d} \rightarrow[0,1]$ be a function of the $C_{0}^{\infty}\left(\mathbb{R}^{d}\right)$ class such that $f(\mathbf{v}) \equiv 1$, when $|\mathbf{v}| \leq \rho / 16$ and $f(\mathbf{v}) \equiv 0$, when $|\mathbf{v}| \geq \rho / 8$. Note that according to Proposition 3.5 we can choose constants $A_{\rho}, C>0$, where $C$ is independent of $\rho$, in such a way that $A_{\rho}<C \rho^{-3}$ (the power three comes from v-derivatives in the right side of (3.33)) and the random sequence

$$
S_{m}^{\mathbf{l}}:=f\left(V\left(\frac{m}{p_{3}}\right)-\mathbf{l}\right)+A_{\rho} \frac{m}{p_{3}}, \quad m \geq 0
$$

is a $\tilde{Q}_{\mathbf{x}, \mathbf{v}}^{(\delta)}$ sub-martingale with respect to the filtration $\left(\mathcal{M}^{m / p_{3}}\right)_{m \geq 0}$ for all $\mathbf{l}$ with $|\mathbf{l}| \in\left(\left(3 M_{*}\right)^{-1}, 3 M_{*}\right)$ provided that $\delta$ is sufficiently small. The restriction on the range of $|\mathbf{l}|$ ensures that the shifted function $f_{\mathbf{l}}(\mathbf{v})=f(\mathbf{v}-\mathbf{l})$ to which we have applied Proposition 3.5 vanishes at $\mathbf{v}=0$. Let $\tilde{Q}_{\mathbf{x}, \mathbf{v}, \pi}^{(\mathrm{r})}$, $\pi \in \mathcal{C}$ denote the family of the regular conditional probability distributions that corresponds to $\tilde{Q}_{\mathbf{x}, \mathbf{v}}^{(\delta)}\left[\cdot \mid \mathcal{M}^{\tau_{n}}\right]$. Then, there exists an $\mathcal{M}^{\tau_{n}}$-measurable, null $\tilde{Q}_{\mathbf{x}, \mathbf{v}}^{(\delta)}$ probability event $Z$ such that for each $\pi \notin Z$ and each $\mathbf{l}$ as above, the random sequence

$$
S_{m, \pi}^{\mathbf{l}}:=S_{m}^{\mathbf{l}} \mathbf{1}_{\left[0, m / p_{3}\right]}\left(\tau_{n}(\pi)\right), \quad m \geq 0
$$

is an $\left(\mathcal{M}^{m / p_{3}}\right)_{m \geq 0}$ sub-martingale under $\tilde{Q}_{\mathbf{x}, \mathbf{v}, \pi}^{(\delta)}$. We can, of course, choose the event $Z$ in such a way that

$$
\tilde{Q}_{\mathbf{x}, \mathbf{v}, \pi}^{(\delta)}\left[T_{n, \pi} \geq \tau_{n}(\pi)\right]=1, \quad \forall \pi \notin Z
$$


where $T_{n, \pi}:=\tau_{n+1} \wedge\left(\tau_{n}(\pi)+\left[p_{3} h\right] / p_{3}\right)$.

Let

$$
\tilde{S}_{m, \pi}:=S_{m, \pi}^{V\left(\tau_{n}(\pi)\right)}=\left[f\left(V\left(\frac{m}{p_{3}}\right)-V\left(\tau_{n}(\pi)\right)\right)+A_{\rho} \frac{m}{p_{3}}\right] \mathbf{1}_{\left[0, m / p_{3}\right]}\left(\tau_{n}(\pi)\right),
$$

then the sub-martingale property of $\left(\tilde{S}_{m, \pi}\right)_{m \geq 0}$ and (3.40) imply that

$$
\tilde{E}_{\mathbf{x}, \mathbf{v}, \pi}^{(\delta)} \tilde{S}_{p_{3} T_{n, \pi}, \pi} \geq \tilde{E}_{\mathbf{x}, \mathbf{v}, \pi}^{(\delta)} \tilde{S}_{p_{3} \tau_{n}(\pi), \pi}=1+A_{\rho} \tau_{n}(\pi) .
$$

In consequence of (3.41) we have

$$
\tilde{E}_{\mathbf{x}, \mathbf{v}, \pi}^{(\delta)}\left[f\left(V\left(T_{n, \pi}\right)-V\left(\tau_{n}(\pi)\right)\right)\right]+A_{\rho} h \geq 1,
$$

as $T_{n, \pi}-\tau_{n}(\pi) \leq h$. From (3.42) we obtain that

$$
A_{\rho} h \geq \tilde{E}_{\mathbf{x}, \mathbf{v}, \pi}^{(\delta)}\left[1-f\left(V\left(T_{n, \pi}\right)-V\left(\tau_{n}(\pi)\right)\right)\right]
$$

so in particular, using the definition of the stopping times $\tau_{n}(\pi)$ and the function $f(\mathbf{v})$, we obtain

$$
\begin{aligned}
A_{\rho} h & \geq \tilde{E}_{\mathbf{x}, \mathbf{v}, \pi}^{(\delta)}\left[1-f\left(V\left(\tau_{n+1}(\pi)\right)-V\left(\tau_{n}(\pi)\right)\right), \tau_{n+1}(\pi) \leq \tau_{n}(\pi)+h\right] \\
& =\tilde{Q}_{\mathbf{x}, \mathbf{v}, \pi}^{(\delta)}\left[\tau_{n+1}(\pi) \leq \tau_{n}(\pi)+h\right] .
\end{aligned}
$$

This proves (3.35).

In order to show that (3.36) also follows let us fix an $h_{0}>0$ such that

$$
\gamma:=e^{-h_{0}}+A_{\rho}\left(1-e^{-h_{0}}\right) h_{0}<1 .
$$

We obtain then

$$
\begin{gathered}
\tilde{E}_{\mathbf{x}, \mathbf{v}}^{(\delta)}\left[\exp \left\{-\left(\tau_{n+1}-\tau_{n}\right)\right\} \mid \mathcal{M}^{\tau_{n}}\right] \leq e^{-h_{0}} \tilde{Q}_{\mathbf{x}, \mathbf{v}}^{(\delta)}\left[\tau_{n+1}-\tau_{n} \geq h_{0} \mid \mathcal{M}^{\tau_{n}}\right]+\tilde{Q}_{\mathbf{x}, \mathbf{v}}^{(\delta)}\left[\tau_{n+1}-\tau_{n} \leq h_{0} \mid \mathcal{M}^{\tau_{n}}\right] \\
\leq e^{-h_{0}}+\left(1-e^{-h_{0}}\right) \tilde{Q}_{\mathbf{x}, \mathbf{v}}^{(\delta)}\left[\tau_{n+1}-\tau_{n} \leq h_{0} \mid \mathcal{M}^{\tau_{n}}\right] \leq e^{-h_{0}}+A_{\rho}\left(1-e^{-h_{0}}\right) h_{0}=\gamma .
\end{gathered}
$$

We used (3.35) in the last step above. From (3.43) one concludes as in Lemma 1.4.5 p. 38 of [8], that (3.36) holds. The proof of Proposition 3.6] is now complete.

\section{The proof of Proposition 3.5.}

The proof of this Proposition follows the blueprint of [1, 4, 5] with the modifications that are necessary to account for the fact that the process with cut-offs may come back to a tube around the past trajectory. As we have mentioned previously, the reason the proof goes through is that the set of such bad times is small: see Proposition 3.3. The rest of the argument is similar, we present it in detail for the convenience of the reader.

Let

$$
\boldsymbol{L}^{(\delta)}(\sigma, s):=\boldsymbol{y}^{(\delta)}(\sigma)+(s-\sigma) \boldsymbol{l}^{(\delta)}(\sigma)
$$

be the linear approximation of the trajectory between times $\sigma$ and $s>\sigma$. We obtain from the definition of the dynamics, see (3.17), that

$$
\left|\boldsymbol{y}^{(\delta)}(s)-\boldsymbol{L}^{(\delta)}(\sigma, s)\right| \leq \frac{\tilde{D}(s-\sigma)^{2}}{2 \sqrt{\delta}}, \quad \delta \in\left(0, \delta_{*}\right] .
$$


In the course of the proof of (3.33) we assume without loss of generality that there exists $k$ such that $t \in\left[t_{k}^{(1)}, t_{k+1}^{(1)}\right)$ and $u \in\left[t_{k}^{(1)}, t_{k+2}^{(1)}\right)$. Throughout this argument we use (4.2) with

$$
\sigma_{s}:=\max \left[s-\delta^{1-\gamma_{A}}, t\right], \quad s \in[t, u]
$$

for some

$$
0<\gamma_{A}<1 / 16
$$

For this choice of $\sigma_{s}$ we have

$$
\left|\boldsymbol{y}^{(\delta)}(s)-\boldsymbol{L}^{(\delta)}\left(\sigma_{s}, s\right)\right| \leq C \delta^{3 / 2-2 \gamma_{A}}, \quad \forall \delta \in(0,1] .
$$

Throughout this section we denote $\hat{\zeta}=\zeta\left(\boldsymbol{y}^{(\delta)}\left(t_{1}\right), \boldsymbol{l}^{(\delta)}\left(t_{1}\right), \ldots, \boldsymbol{y}^{(\delta)}\left(t_{n}\right), \boldsymbol{l}^{(\delta)}\left(t_{n}\right)\right)$. We also first assume that the test function $G \in C_{b}^{2}\left(\mathbb{R}_{*}^{2}\right)$ as we will use the Taylor formula repeatedly. Note that, according to (3.17),

$$
G\left(\boldsymbol{l}^{(\delta)}(u)\right)-G\left(\boldsymbol{l}^{(\delta)}(t)\right)=-\frac{1}{\sqrt{\delta}} \sum_{j=1}^{2} \int_{t}^{u} \partial_{j} G\left(\boldsymbol{l}^{(\delta)}(s)\right) F_{j, \delta}\left(s, \frac{\boldsymbol{y}^{(\delta)}(s)}{\delta}, \boldsymbol{l}^{(\delta)}(s)\right) d s .
$$

Once again, using (3.17) and the Taylor formula between the times $\sigma_{s}$ and $s$ we can rewrite then (4.6) in the form $I^{(1)}+I^{(2)}+I^{(3)}$, where

$$
\begin{aligned}
I^{(1)} & :=-\frac{1}{\sqrt{\delta}} \sum_{j=1}^{2} \int_{t}^{u} \partial_{j} G\left(\boldsymbol{l}^{(\delta)}\left(\sigma_{s}\right)\right) F_{j, \delta}\left(s, \frac{\boldsymbol{y}^{(\delta)}(s)}{\delta}, \boldsymbol{l}^{(\delta)}\left(\sigma_{s}\right)\right) d s \\
I^{(2)} & :=\frac{1}{\delta} \sum_{i, j=1}^{2} \int_{t}^{u} d s \int_{\sigma_{s}}^{s} \partial_{j} G\left(\boldsymbol{l}^{(\delta)}(\rho)\right) \partial_{\ell_{i}} F_{j, \delta}\left(s, \frac{\boldsymbol{y}^{(\delta)}(s)}{\delta}, \boldsymbol{l}^{(\delta)}(\rho)\right) F_{i, \delta}\left(\rho, \frac{\boldsymbol{y}^{(\delta)}(\rho)}{\delta}, \boldsymbol{l}^{(\delta)}(\rho)\right) d \rho, \\
I^{(3)} & :=\frac{1}{\delta} \sum_{i, j=1}^{2} \int_{t}^{u} d s \int_{\sigma_{s}}^{s} \partial_{i, j}^{2} G\left(\boldsymbol{l}^{(\delta)}(\rho)\right) F_{j, \delta}\left(s, \frac{\boldsymbol{y}^{(\delta)}(s)}{\delta}, \boldsymbol{l}^{(\delta)}(\rho)\right) F_{i, \delta}\left(\rho, \frac{\boldsymbol{y}^{(\delta)}(\rho)}{\delta}, \boldsymbol{l}^{(\delta)}(\rho)\right) d \rho
\end{aligned}
$$

and $\sigma_{s}$ is given by (4.3). The following lemma estimates the three terms above.

Lemma 4.1 Suppose that $(\mathbf{x}, \mathbf{v}), \zeta, t_{1}, \ldots, t_{n}$ and $\epsilon_{i}, i=1, \ldots, 8$ are as in the statement of Proposition 3.5. Then, there exists a constant $C>0$ such that for any function $G \in C_{b}^{2}\left([0, T] \times \mathbb{R}_{*}^{4}\right)$ we have

$$
\begin{aligned}
& \left|\mathbb{E}\left\{\left[I^{(1)}-\sum_{j=1}^{2} \int_{t}^{u} E_{j}\left(\boldsymbol{l}^{(\delta)}\left(\sigma_{s}\right)\right) \bar{\Theta}^{2}(s) \partial_{j} G\left(\boldsymbol{l}^{(\delta)}\left(\sigma_{s}\right)\right) d s\right] \hat{\zeta}\right\}\right| \leq C \delta^{1 / 6}\left[(u-t) \vee \frac{1}{p_{3}}\right]\|G\|_{1} \mathbb{E} \hat{\zeta}, \quad(4 . ') \\
& \left|\mathbb{E}\left\{\left[I^{(2)}-\sum_{j=1}^{2} \int_{t}^{u} J_{j}\left(s ; \boldsymbol{y}^{(\delta)}(\cdot), \boldsymbol{l}^{(\delta)}(\cdot)\right) \bar{\Theta}(s) \partial_{j} G\left(\boldsymbol{l}^{(\delta)}(s)\right) d s\right] \hat{\zeta}\right\}\right| \leq C \delta^{1 / 6}\left[(u-t) \vee \frac{1}{p_{3}}\right]\|G\|_{1} \mathbb{E} \hat{\zeta}
\end{aligned}
$$

and

$$
\left|\mathbb{E}\left[I^{(3)}-\sum_{i, j=1}^{2} \int_{t}^{u} D_{i, j}\left(\boldsymbol{l}^{(\delta)}(s)\right) \bar{\Theta}^{2}(s) \partial_{i, j}^{2} G\left(\boldsymbol{l}^{(\delta)}(s)\right) d s\right] \hat{\zeta}\right| \leq C \delta^{1 / 6}\left[(u-t) \vee \frac{1}{p_{3}}\right]\|G\|_{3} \mathbb{E} \hat{\zeta},
$$


with

$$
J_{j}\left(s ; \boldsymbol{y}^{(\delta)}(\cdot), \boldsymbol{l}^{(\delta)}(\cdot)\right):=-\sum_{i=1}^{d} \bar{\Theta}_{i}(s) D_{i, j}\left(\boldsymbol{l}^{(\delta)}(s)\right), \quad \bar{\Theta}_{i}(s):=\partial_{l_{i}} \Theta\left(s, \boldsymbol{y}^{(\delta)}(s), \boldsymbol{l}^{(\delta)}(s) ; \boldsymbol{y}^{(\delta)}(\cdot), \boldsymbol{l}^{(\delta)}(\cdot)\right),
$$

and $\bar{\Theta}(s):=\Theta\left(s, \boldsymbol{y}^{(\delta)}(s), \boldsymbol{l}^{(\delta)}(s) ; \boldsymbol{y}^{(\delta)}(\cdot), \boldsymbol{l}^{(\delta)}(\cdot)\right)$. The choice of the constants $\gamma, C$ does not depend on $(\mathbf{x}, \mathbf{v}), \delta \in(0,1], \zeta$, times $t_{1}, \ldots, t_{n}, u, t$, or the function $G$.

\subsection{The proof of (4.7)}

Using the linear approximation (4.1), the term $I^{(1)}$ can be rewritten in the form $J^{(1)}+J^{(2)}$, where

$$
J^{(1)}:=-\frac{1}{\sqrt{\delta}} \sum_{j=1}^{2} \int_{t}^{u} \partial_{j} G\left(\boldsymbol{l}^{(\delta)}\left(\sigma_{s}\right)\right) F_{j, \delta}\left(s, \frac{\boldsymbol{L}^{(\delta)}\left(\sigma_{s}, s\right)}{\delta}, \boldsymbol{l}^{(\delta)}\left(\sigma_{s}\right)\right) d s
$$

and

$$
J^{(2)}:=-\frac{1}{\delta^{3 / 2}} \sum_{i, j=1}^{2} \int_{t}^{u} \int_{0}^{1} \partial_{j} G\left(\boldsymbol{l}^{(\delta)}\left(\sigma_{s}\right)\right) \partial_{y_{i}} F_{j, \delta}\left(s, \frac{\boldsymbol{R}^{(\delta)}(\theta, \sigma, s)}{\delta}, \boldsymbol{l}^{(\delta)}\left(\sigma_{s}\right)\right)\left(y_{i}^{(\delta)}(s)-L_{i}^{(\delta)}\left(\sigma_{s}, s\right)\right) d s d \theta,
$$

where $\boldsymbol{R}^{(\delta)}\left(\theta, \sigma_{s}, s\right)=(1-\theta) \boldsymbol{L}\left(\sigma_{s}, s\right)+\theta \boldsymbol{y}^{(\delta)}(s)$.

\subsubsection{The estimate for $J^{(1)}$}

We will show that $J^{(1)}$ becomes small as $\delta \downarrow 0$, namely

$$
\left|\mathbb{E}\left[J^{(1)} \hat{\zeta}\right]\right| \leq C \delta^{1 / 6}\left[(u-t) \vee \frac{1}{p_{3}}\right]\|G\|_{1} \mathbb{E} \hat{\zeta} .
$$

To see this we shall further split $J^{(1)}=J_{A}^{(1)}+J_{B}^{(1)}$. The first term contains integration over the "bad" times when the point $\boldsymbol{y}^{(\delta)}\left(\sigma_{s}\right)$ is inside the tube around the past, while the second contains integration over the good times. That is, we define

$$
J_{A}^{(1)}:=-\frac{1}{\sqrt{\delta}} \sum_{j=1}^{2} \int_{t}^{u} \mathbf{1}_{\mathfrak{X}_{\delta}\left(k-1, p_{4}\right)}\left(\boldsymbol{y}^{(\delta)}\left(\sigma_{s}\right)\right) \partial_{j} G\left(\boldsymbol{l}^{(\delta)}\left(\sigma_{s}\right) F_{j, \delta}\left(s, \frac{\boldsymbol{L}^{(\delta)}\left(\sigma_{s}, s\right)}{\delta}, \boldsymbol{l}^{(\delta)}\left(\sigma_{s}\right),\right) d s\right.
$$

and

$$
J_{B}^{(1)}:=-\frac{1}{\sqrt{\delta}} \sum_{j=1}^{2} \int_{t}^{u} \mathbf{1}_{\mathfrak{X}_{\delta}^{c}\left(k-1, p_{4}\right)}\left(\boldsymbol{y}^{(\delta)}\left(\sigma_{s}\right)\right) \partial_{j} G\left(\boldsymbol{l}^{(\delta)}\left(\sigma_{s}\right)\right) F_{j, \delta}\left(s, \frac{\boldsymbol{L}^{(\delta)}\left(\sigma_{s}, s\right)}{\delta}, \boldsymbol{l}^{(\delta)}\left(\sigma_{s}\right)\right) d s .
$$

Here $\mathfrak{X}_{\delta}\left(k-1, p_{4}\right)$ denotes the tube defined in (3.23), and $\mathfrak{X}_{\delta}^{c}\left(k-1, p_{4}\right)$ is its complement. Note that if $\boldsymbol{y}^{(\delta)}\left(\sigma_{s}\right) \in \mathfrak{X}_{\delta}\left(k-1, p_{4}\right)$ then, since $\left|\boldsymbol{y}^{(\delta)}\left(\sigma_{s}\right)-\boldsymbol{y}^{(\delta)}(s)\right| \leq 2 M_{*} \delta^{1-\gamma_{A}}$, we have $\boldsymbol{y}^{(\delta)}(s) \in \mathfrak{X}_{\delta}\left(k-1,2 p_{4}\right)$, provided that $\delta>0$ is sufficiently small. Recall here that for $\epsilon_{4}$, defined in (3.1), the assumptions of Proposition 3.5 guarantee that $\gamma_{A}<1 / 16<1-\epsilon_{4}$. Hence, we can estimate the contribution of $J_{A}^{(1)}$ to (3.33) using Proposition 3.3 as

$$
\begin{aligned}
\left|\mathbb{E}\left[J_{A}^{(1)} \hat{\zeta}\right]\right| & \leq \frac{1}{\sqrt{\delta}} \sum_{j=1}^{2} \mathbb{E}\left[\hat{\zeta} \int_{t}^{u} \mathbf{1}_{A_{\delta}\left(k, 2 p_{4}\right)}(s)\left|\partial_{j} G\left(\boldsymbol{l}^{(\delta)}\left(\sigma_{s}\right)\right) F_{j, \delta}\left(s, \frac{\boldsymbol{L}^{(\delta)}\left(\sigma_{s}, s\right)}{\delta}, \boldsymbol{l}^{(\delta)}\left(\sigma_{s}\right)\right)\right| d s\right] \\
& \leq \frac{C p_{2}^{2}}{\sqrt{\delta} p_{1} p_{4}} N_{4}^{1 / 2}\|G\|_{1} \mathbb{E} \hat{\zeta} \leq C \delta^{1 / 6}\left[(u-t) \vee \frac{1}{p_{3}}\right]\|G\|_{1} \mathbb{E} \hat{\zeta}
\end{aligned}
$$


provided that

$$
\frac{1}{6} \leq \epsilon_{4}-\frac{1}{2}-2\left(\epsilon_{1}+\epsilon_{2}\right)-\epsilon_{3}-\frac{\epsilon_{8}}{2},
$$

which is true under the assumptions of Proposition 3.5 (see (3.1) for the definition of $\epsilon_{j}, j=1, \ldots, 8$ ). We note that while Proposition 3.3 does not allow to control the time spent inside the tube in a non-transversal direction, that is, when the cut-off $\Theta_{\delta}=0$, such times do not contribute to $J_{A}^{(1)}$ as then the integrand $F_{j, \delta}$ is automatically equal to zero.

Now we will proceed with the estimate of $\left|\mathbb{E}\left[J_{B}^{(1)} \hat{\zeta}\right]\right|$. This will be done with the help of the mixing Lemma 3.4 Suppose that $\left[t_{l}^{(2)}, t_{l+1}^{(2)}\right)$ are the intervals of the finer mesh contained in the interval $[t, u]:\left[t_{l}^{(2)}, t_{l+1}^{(2)}\right) \subseteq[t, u]$ for $l_{1} \leq l \leq l_{2}$ and that $t_{l_{1}-1}^{(2)} \leq t$, and $t_{l_{2}+1}^{(2)} \geq u$. There are at most $2\left(p_{2} / p_{1}+1\right)=2\left(\left[\delta^{-\epsilon_{2}}\right]+1\right)$ of such intervals as $|u-t| \leq 2 / p_{1}$. In order to use mixing we will need $\sigma_{s}$ and $s$ to lie inside the same interval of such type. Hence we set

$$
G_{J}=\left[s: s \in\left[t_{l}^{(2)}, t_{l+1}^{(2)}\right), \quad t \leq s \leq u, \quad s \geq t_{l}^{(2)}+\delta^{1-\gamma_{A}}\right] \cap\left[s: \quad s \geq t+\delta^{1-\gamma_{A}}\right]
$$

and denote by $G_{J}^{c}$ its complement in $[t, u]$. Observe that

$$
\left|G_{J}^{c}\right| \leq \frac{C p_{2} \delta^{1-\gamma_{A}}}{p_{1}}
$$

Let $s \in G_{J}$ - we will use part (i) of Lemma 3.4 with $\tilde{X}_{1}(\mathbf{x}):=-\partial_{x_{j}} H(\mathbf{x}), \tilde{X}_{2}(\mathbf{x}) \equiv 1$,

$$
Z:=\mathbf{1}_{\mathfrak{X}_{\delta}^{c}\left(k-1, p_{4}\right)}\left(\boldsymbol{y}^{(\delta)}\left(\sigma_{s}\right)\right) \Theta\left(\sigma_{s}, \boldsymbol{L}^{(\delta)}\left(\sigma_{s}, s\right), \boldsymbol{l}^{(\delta)}\left(\sigma_{s}\right)\right) \partial_{j} G\left(\boldsymbol{l}^{(\delta)}\left(\sigma_{s}\right)\right) \hat{\zeta}
$$

and $g_{1}:=\boldsymbol{L}^{(\delta)}\left(\sigma_{s}, s\right) \delta^{-1}$. We have replaced $s$ in the argument of $\Theta$ by $\sigma_{s}$ since $s$ and $\sigma_{s}$ both lie inside $\left[t_{l}^{(2)}, t_{l+1}^{(2)}\right)$, and $\Theta$ does not vary in $s$ on such intervals. Note that $g_{1}$ and $Z$ are both $\mathcal{F}_{\sigma_{s}}$ measurable. We need to verify that (3.29) holds, that is, that

$$
\left|g_{1}-\frac{\boldsymbol{y}^{(\delta)}(\rho)}{\delta}\right|=\frac{1}{\delta}\left|\boldsymbol{L}^{(\delta)}\left(\sigma_{s}, s\right)-\boldsymbol{y}^{(\delta)}(\rho)\right| \geq \frac{r}{\delta},
$$

for all $0 \leq \rho \leq \sigma_{s}$. To this end suppose that $Z \neq 0$. Assume first that $\rho \in\left[0, t_{k-1}^{(1)}\right]-$ then we use the fact that $\boldsymbol{y}^{(\delta)}\left(\sigma_{s}\right)$ is not in the tube $\mathfrak{X}_{\delta}\left(k-1, p_{4}\right)$. More precisely, since $\left|\boldsymbol{y}^{(\delta)}\left(\sigma_{s}\right)-\boldsymbol{y}^{(\delta)}(\rho)\right| \geq 2 / p_{4}$, we have

$$
\left|g_{1}-\frac{\boldsymbol{y}^{(\delta)}(\rho)}{\delta}\right|=\frac{1}{\delta}\left|\boldsymbol{L}^{(\delta)}\left(\sigma_{s}, s\right)-\boldsymbol{y}^{(\delta)}(\rho)\right| \geq \frac{1}{2 p_{4} \delta},
$$

because of (4.5), provided that $C \delta^{3 / 2-\gamma_{A}}<1 / p_{4}$. The latter condition holds for a sufficiently small $\delta>0$ because $3 / 2-\gamma_{A}>1>\epsilon_{4}-$ see (4.4). For $\rho \in\left[t_{k-1}^{(1)}, \sigma_{s}\right]$ we use the cut-offs that "propel the trajectory forward". We consider two cases. First, if $\sigma_{s} \leq t_{k+1}^{(1)}$ and $\delta$ is sufficiently small we have, using (3.19)

$$
\begin{aligned}
& \left(\boldsymbol{L}^{(\delta)}\left(\sigma_{s}, s\right)-\boldsymbol{y}^{(\delta)}(\rho)\right) \cdot \hat{\boldsymbol{l}}^{(\delta)}\left(t_{k-1}^{(1)}\right) \geq\left(s-\sigma_{s}\right) \boldsymbol{l}^{(\delta)}\left(\sigma_{s}\right) \cdot \hat{\boldsymbol{l}}^{(\delta)}\left(t_{k-1}^{(1)}\right) \\
& +\int_{\rho}^{\sigma_{s}} \boldsymbol{l}^{(\delta)}\left(\rho_{1}\right) \cdot \hat{\boldsymbol{l}}^{(\delta)}\left(t_{k-1}^{(1)}\right) d \rho_{1} \geq \frac{s-\sigma_{s}}{2 M_{*}}\left(1-\frac{2}{N_{1}}\right) .
\end{aligned}
$$


When, on the other hand $\sigma_{s}>t_{k+1}^{(1)}$ we obtain using (3.20) that the left hand side of (4.17) is greater than, or equal to $\left(2 M_{*}\right)^{-1}\left(s-\sigma_{s}\right)\left(1-18 / N_{1}\right)$. We see that in both of those two cases condition (3.29) is satisfied with $r=\left(1-18 / N_{1}\right)\left(s-\tilde{\sigma}_{s}\right) /\left(2 M_{*}\right)=C \delta^{-\gamma_{A}}$. Using Lemma 3.4 we estimate the first term comes from $s \in G_{J}$ and is bounded using mixing, and the second arises from $s \in G_{J}^{c}$ and is controlled by (4.15):

$$
\left|\mathbb{E}\left[J_{B}^{(1)} \hat{\zeta}\right]\right| \leq \frac{\tilde{D}}{\sqrt{\delta}}\|G\|_{1} \mathbb{E}[\hat{\zeta}] \phi\left(C \delta^{-\gamma_{A}}\right)\left|G_{J}\right|+\frac{\tilde{D}}{\sqrt{\delta}}\|G\|_{1} \mathbb{E}[\hat{\zeta}]\left|G_{J}^{c}\right| .
$$

The first term above decays faster than any power of $\delta$ because of (2.3), while the second may be bounded using (4.15):

$$
\left|\mathbb{E}\left[J_{B}^{(1)} \hat{\zeta}\right]\right| \leq \frac{C p_{2}}{p_{1} \delta^{1 / 2}}\|G\|_{1} \mathbb{E}[\hat{\zeta}] \delta^{1-\gamma_{A}} \leq C\|G\|_{1} \mathbb{E}[\hat{\zeta}] \delta^{1 / 6}\left[(u-t) \vee \frac{1}{p_{3}}\right]
$$

for

$$
\frac{1}{6} \leq \frac{1}{2}-\gamma_{A}-\epsilon_{1}-\epsilon_{2}-\epsilon_{3}
$$

Together, (4.12) and (4.18) imply (4.11). This concludes the estimate for $J^{(1)}$.

\subsubsection{The estimate for $J^{(2)}$}

The term $J^{(2)}$ defined by (4.10) produces a non-trivial contribution in the limit $\delta \downarrow 0$. In order to find its asymptotic behavior we write it as $J^{(2)}=J_{1}^{(2)}+J_{2}^{(2)}$, where

$$
J_{1}^{(2)}:=-\frac{1}{\delta^{3 / 2}} \sum_{i, j=1}^{2} \int_{t}^{u} \partial_{j} G\left(\boldsymbol{l}^{(\delta)}\left(\sigma_{s}\right)\right) \partial_{y_{i}} F_{j, \delta}\left(s, \frac{\boldsymbol{L}^{(\delta)}\left(\sigma_{s}, s\right)}{\delta}, \boldsymbol{l}^{(\delta)}\left(\sigma_{s}\right)\right)\left(y_{i}^{(\delta)}(s)-L_{i}^{(\delta)}\left(\sigma_{s}, s\right)\right) d s
$$

and

$$
\begin{aligned}
J_{2}^{(2)}:= & -\frac{1}{\delta^{5 / 2}} \sum_{i, j, k=1}^{2} \int_{t}^{u} d s \int_{0}^{1} \int_{0}^{1} \partial_{y_{i}, y_{k}}^{2} F_{j, \delta}\left(s, \frac{\boldsymbol{R}^{(\delta)}\left(\theta v, \sigma_{s}, s\right)}{\delta}, \boldsymbol{l}^{(\delta)}\left(\sigma_{s}\right)\right) v \\
& \times \partial_{j} G\left(\boldsymbol{l}^{(\delta)}\left(\sigma_{s}\right)\right)\left(y_{i}^{(\delta)}(s)-L_{i}^{(\delta)}\left(\sigma_{s}, s\right)\right)\left(y_{k}^{(\delta)}(s)-L_{k}^{(\delta)}\left(\sigma_{s}, s\right)\right) d v d \theta
\end{aligned}
$$

The term involving $J_{2}^{(2)}$ may be handled with the help of (3.14) with $\beta_{2}=0$ and (4.5). We obtain

$$
\begin{gathered}
\left|\mathbb{E}\left[J_{2}^{(2)} \hat{\zeta}\right]\right| \leq C \tilde{D}\|G\|_{1}(u-t) \delta^{-5 / 2} \delta^{3-4 \gamma_{A}} \mathbb{E} \hat{\zeta} \\
\leq C \delta^{1 / 2-4 \gamma_{A}}(u-t)\|G\|_{1} \mathbb{E} \hat{\zeta} \leq C \delta^{1 / 6}(u-t)\|G\|_{1} \mathbb{E} \hat{\zeta}
\end{gathered}
$$

because, according to (4.4),$\gamma_{A}<1 / 16$. Hence, $J_{2}^{(2)}$ makes no contribution to the limit.

In order to estimate the term corresponding to $J_{1}^{(2)}$ we write

$$
\begin{aligned}
J_{1}^{(2)} & :=-\frac{1}{\delta^{3 / 2}} \sum_{i, j=1}^{2} \int_{t}^{u} d s \int_{\sigma_{s}}^{s} \partial_{j} G\left(\boldsymbol{l}^{(\delta)}\left(\sigma_{s}\right)\right) \partial_{y_{i}} F_{j, \delta}\left(s, \frac{\boldsymbol{L}^{(\delta)}\left(\sigma_{s}, s\right)}{\delta}, \boldsymbol{l}^{(\delta)}\left(\sigma_{s}\right)\right)\left(s-\rho_{1}\right) i_{i}^{(\delta)}\left(\rho_{1}\right) d \rho_{1} \\
& =\frac{1}{\delta^{2}} \sum_{i, j=1}^{2} \int_{t}^{u} d s \int_{\sigma_{s}}^{s} \partial_{j} G\left(\boldsymbol{l}^{(\delta)}\left(\sigma_{s}\right)\right) \partial_{y_{i}} F_{j, \delta}\left(s, \frac{\boldsymbol{L}^{(\delta)}\left(\sigma_{s}, s\right)}{\delta}, \boldsymbol{l}^{(\delta)}\left(\sigma_{s}\right)\right) \\
& \times\left(s-\rho_{1}\right) F_{i, \delta}\left(\rho_{1}, \frac{\boldsymbol{y}^{(\delta)}\left(\rho_{1}\right)}{\delta}, \boldsymbol{l}^{(\delta)}\left(\sigma_{s}\right)\right) d \rho_{1} .
\end{aligned}
$$


An application of (4.5), definition (3.16) and Lemma 3.1] as in the estimate for $J_{2}^{(2)}$ yields

$$
\begin{aligned}
& \mid \mathbb{E}\left[J_{1}^{(2)} \zeta\right]-\frac{1}{\delta^{2}} \sum_{i, j=1}^{2} \int_{t}^{u} d s \int_{\sigma_{s}}^{s}\left(s-\rho_{1}\right) \mathbb{E}\left[\partial_{j} G\left(\boldsymbol{l}^{(\delta)}\left(\sigma_{s}\right)\right) \partial_{y_{i}} F_{j, \delta}\left(s, \frac{\boldsymbol{L}^{(\delta)}\left(\sigma_{s}, s\right)}{\delta}, \boldsymbol{l}^{(\delta)}\left(\sigma_{s}\right)\right)\right. \\
& \left.\times F_{i, \delta}\left(\rho_{1}, \frac{\boldsymbol{L}^{(\delta)}\left(\sigma_{s}, \rho_{1}\right)}{\delta}, \boldsymbol{l}^{(\delta)}\left(\sigma_{s}\right)\right) \hat{\zeta}\right] d \rho_{1} \mid \leq C \delta^{1 / 2-4 \gamma_{A}}(u-t)\|G\|_{1} \mathbb{E} \hat{\zeta} \leq C \delta^{1 / 6}(u-t)\|G\|_{1} \mathbb{E} \hat{\zeta}
\end{aligned}
$$

The second term on the left side of (4.23) can be written as a sum $K_{A}+K_{B}+K_{C}$, where the first term accounts for the time inside the tube:

$$
\begin{aligned}
K_{A}:= & \frac{1}{\delta^{2}} \sum_{i, j=1}^{2} \int_{t}^{u} d s \int_{\sigma_{s}}^{s}\left(s-\rho_{1}\right) \mathbb{E}\left[\mathbf{1}_{\mathfrak{X}\left(k-1, p_{4}\right)}\left(\boldsymbol{y}^{(\delta)}\left(\sigma_{s}\right)\right) \partial_{j} G\left(\boldsymbol{l}^{(\delta)}\left(\sigma_{s}\right)\right)\right. \\
& \left.\times \partial_{y_{i}} F_{j, \delta}\left(s, \frac{\boldsymbol{L}^{(\delta)}\left(\sigma_{s}, s\right)}{\delta}, \boldsymbol{l}^{(\delta)}\left(\sigma_{s}\right)\right) F_{i, \delta}\left(\rho_{1}, \frac{\boldsymbol{L}^{(\delta)}\left(\sigma_{s}, \rho_{1}\right)}{\delta}, \boldsymbol{l}^{(\delta)}\left(\sigma_{s}\right)\right) \hat{\zeta}\right] d \rho_{1},
\end{aligned}
$$

while the other two concern the good times when $\boldsymbol{y}^{(\delta)}\left(\sigma_{s}\right)$ is outside the tube

$$
\begin{gathered}
K_{B}:=\frac{1}{\delta^{2}} \sum_{i, j=1}^{2} \int_{t}^{u} d s \int_{\sigma_{s}}^{s}\left(s-\rho_{1}\right) \mathbb{E}\left[\mathbf{1}_{\mathfrak{X}^{c}\left(k-1, p_{4}\right)}\left(\boldsymbol{y}^{(\delta)}\left(\sigma_{s}\right)\right) \partial_{j} G\left(\boldsymbol{l}^{(\delta)}\left(\sigma_{s}\right)\right) \partial_{y_{i}} \Theta_{\delta}\left(s, \frac{\boldsymbol{L}^{(\delta)}\left(\sigma_{s}, s\right)}{\delta}, \boldsymbol{l}^{(\delta)}\left(\sigma_{s}\right)\right)\right. \\
\left.\times \partial_{y_{j}} H\left(\frac{\boldsymbol{L}^{(\delta)}\left(\sigma_{s}, s\right)}{\delta}\right) F_{i, \delta}\left(\rho_{1}, \frac{\boldsymbol{L}^{(\delta)}\left(\sigma_{s}, \rho_{1}\right)}{\delta}, \boldsymbol{l}^{(\delta)}\left(\sigma_{s}\right)\right) \hat{\zeta}\right] d \rho_{1} .
\end{gathered}
$$

and

$$
\begin{aligned}
K_{C}:= & \frac{1}{\delta^{2}} \sum_{i, j=1}^{2} \int_{t}^{u} d s \int_{\sigma_{s}}^{s}\left(s-\rho_{1}\right) \mathbb{E}\left[\mathbf{1}_{\mathfrak{X}^{c}\left(k-1, p_{4}\right)}\left(\boldsymbol{y}^{(\delta)}\left(\sigma_{s}\right)\right) \partial_{j} G\left(\boldsymbol{l}^{(\delta)}\left(\sigma_{s}\right)\right) \Theta\left(s, \boldsymbol{L}^{(\delta)}\left(\sigma_{s}, s\right), \boldsymbol{l}^{(\delta)}\left(\sigma_{s}\right)\right)\right. \\
& \left.\times \Theta\left(\rho_{1}, \boldsymbol{L}^{(\delta)}\left(\sigma_{s}, \rho_{1}\right), \boldsymbol{l}^{(\delta)}\left(\sigma_{s}\right)\right) \partial_{y_{i} y_{j}}^{2} H\left(\frac{\boldsymbol{L}^{(\delta)}\left(\sigma_{s}, s\right)}{\delta}\right) \partial_{y_{i}} H\left(\frac{\boldsymbol{L}^{(\delta)}\left(\sigma_{s}, \rho_{1}\right)}{\delta}\right) \hat{\zeta}\right] d \rho_{1} \cdot(4.24)
\end{aligned}
$$

By virtue of Proposition 3.3 we obtain that $K_{A}$ may be bounded by

$$
\left|K_{A}\right| \leq C \delta^{-2 \gamma_{A}} \tilde{D}^{2}\|G\|_{1} \frac{p_{2}^{2} N_{4}^{1 / 2}}{p_{1} p_{4}} \mathbb{E} \hat{\zeta} \leq C \delta^{1 / 6}\left[(u-t) \vee \frac{1}{p_{3}}\right]\|G\|_{1} \mathbb{E} \hat{\zeta},
$$

because

$$
\frac{1}{6} \leq \epsilon_{4}-2\left(\gamma_{A}+\epsilon_{1}+\epsilon_{2}\right)-\epsilon_{3}-\frac{\epsilon_{8}}{2} .
$$

The term $K_{B}$ that involves differentiating the cut-off function can be estimated with the help of the first inequality in (3.14) by

$$
\left|K_{B}\right| \leq C \delta^{1-2\left(\gamma_{A}+\epsilon_{1}+\epsilon_{2}\right)}(u-t)\|G\|_{1} \mathbb{E} \hat{\zeta} \leq C \delta^{1 / 6}(u-t)\|G\|_{1} \mathbb{E} \hat{\zeta}
$$

as

$$
\frac{1}{6} \leq 1-2\left(\gamma_{A}+\epsilon_{1}+\epsilon_{2}\right)
$$


To deal with the term $K_{C}$ that turns out to be the principal contribution to $I^{(1)}$ we first observe that $\rho_{1}$, as the first argument in the function $\Theta$ on the second line in (4.24), may be replaced by $s$, as long as $s$ and $\sigma_{s}$ lie in the same interval of the $1 / p_{2}$-mesh - that is, for $s \in G_{J}$, see (4.14). As the measure of the set $\left|G_{J}^{c}\right|$ is bounded as in 4.15), we have

$$
\left|K_{C}-K_{C}^{\prime}\right| \leq C \delta^{1-3 \gamma_{A}-\epsilon_{2}}\left|G\left\|_{1} \mathbb{E} \hat{\zeta} \leq C \delta^{1 / 6}\left[(u-t) \vee \frac{1}{p_{3}}\right] \mid G\right\|_{1} \mathbb{E} \hat{\zeta}\right.
$$

where

$$
\begin{aligned}
K_{C}^{\prime}:= & \frac{1}{\delta^{2}} \sum_{i, j=1}^{2} \int_{t}^{u} d s \int_{\sigma_{s}}^{s}\left(s-\rho_{1}\right) \mathbb{E}\left[\mathbf{1}_{\mathfrak{X}^{c}\left(k-1, p_{4}\right)}\left(\boldsymbol{y}^{(\delta)}\left(\sigma_{s}\right)\right) \partial_{j} G\left(\boldsymbol{l}^{(\delta)}\left(\sigma_{s}\right)\right) \Theta\left(s, \boldsymbol{L}^{(\delta)}\left(\sigma_{s}, s\right), \boldsymbol{l}^{(\delta)}\left(\sigma_{s}\right)\right)\right. \\
& \left.\times \Theta\left(s, \boldsymbol{L}^{(\delta)}\left(\sigma_{s}, \rho_{1}\right), \boldsymbol{l}^{(\delta)}\left(\sigma_{s}\right)\right) \partial_{y_{i} y_{j}}^{2} H\left(\frac{\boldsymbol{L}^{(\delta)}\left(\sigma_{s}, s\right)}{\delta}\right) \partial_{y_{i}} H\left(\frac{\boldsymbol{L}^{(\delta)}\left(\sigma_{s}, \rho_{1}\right)}{\delta}\right) \hat{\zeta}\right] d \rho_{1} .
\end{aligned}
$$

We introduce some auxiliary notation. For $j=1,2$ we let $V_{j}\left(\mathbf{y}, \mathbf{y}^{\prime}, \mathbf{l}\right):=\Delta R_{j}\left(\mathbf{y}-\mathbf{y}^{\prime}\right)$ - the notation here is as in 2.10). We let also

$$
\begin{aligned}
& \Lambda\left(t, \mathbf{y}, \mathbf{y}^{\prime}, \mathbf{l} ; \pi\right):=\Theta(t, \mathbf{y}, \mathbf{l} ; \pi) \Theta\left(t, \mathbf{y}^{\prime}, \mathbf{l} ; \pi\right), \quad t \geq 0, \mathbf{y}, \mathbf{y}^{\prime} \in \mathbb{R}^{2}, \mathbf{l} \in \mathbb{R}_{*}^{2}, \pi \in \mathcal{C}, \\
& P:=\left(\boldsymbol{L}^{(\delta)}\left(\sigma_{s}, s\right), \boldsymbol{L}^{(\delta)}\left(\sigma_{s}, \rho_{1}\right), \boldsymbol{l}^{(\delta)}\left(\sigma_{s}\right)\right), \quad P_{\delta}:=\left(\delta^{-1} \boldsymbol{L}^{(\delta)}\left(\sigma_{s}, s\right), \delta^{-1} \boldsymbol{L}^{(\delta)}\left(\sigma_{s}, \rho_{1}\right), \boldsymbol{l}^{(\delta)}\left(\sigma_{s}\right)\right)
\end{aligned}
$$

and $\bar{\Theta}(s):=\Theta\left(s, \boldsymbol{y}^{(\delta)}(s), \boldsymbol{l}^{(\delta)}(s) ; \boldsymbol{y}^{(\delta)}(\cdot), \boldsymbol{l}^{(\delta)}(\cdot)\right)$. Now the argument used to estimate $\left|\mathbb{E}\left[J_{B}^{(1)} \hat{\zeta}\right]\right|$ (cf. the calculations in (4.16)-4.17) and the respective explanations) can be invoked. We use part (ii) of Lemma 3.4 for $s \in G_{J}$ with

$$
\begin{aligned}
& Z=\mathbf{1}_{\mathfrak{X}_{\delta}^{c}\left(k-1, p_{4}\right)}\left(\boldsymbol{y}^{(\delta)}\left(\sigma_{s}\right)\right) \Lambda\left(\sigma_{s}, P\right) \partial_{j} G\left(\boldsymbol{l}^{(\delta)}\left(\sigma_{s}\right)\right) \hat{\zeta} \\
& g_{1}:=\delta^{-1} \boldsymbol{L}^{(\delta)}\left(\sigma_{s}, s\right), \quad g_{2}:=\delta^{-1} \boldsymbol{L}^{(\delta)}\left(\sigma_{s}, \rho_{1}\right), \\
& r=\left(1-\frac{18}{N}\right) \times \frac{\rho_{1}-\sigma_{s}}{2 M_{*}}, \quad r_{1}=\left(1-\frac{18}{N}\right) \times \frac{s-\rho_{1}}{2 M_{*}} .
\end{aligned}
$$

Now, for $s \in G_{J}$ we have, using (3.32)

$$
\begin{aligned}
& \left|\sum_{i=1}^{2} \mathbb{E}\left[Z \partial_{y_{i} y_{j}}^{2} H\left(\frac{\boldsymbol{L}^{(\delta)}\left(\sigma_{s}, s\right)}{\delta}\right) \partial_{y_{i}} H\left(\frac{\boldsymbol{L}^{(\delta)}\left(\sigma_{s}, \rho_{1}\right)}{\delta}\right)\right]+\mathbb{E}\left[Z V_{j}\left(P_{\delta}\right)\right]\right| \\
& \leq C \phi^{1 / 2}\left(C \frac{s-\rho_{1}}{\delta}\right) \phi^{1 / 2}\left(C \frac{\rho_{1}-\sigma_{s}}{\delta}\right) .
\end{aligned}
$$

Hence, we obtain, estimating the integral over the times $s \in G_{J}^{c}$ in the usual manner:

$$
\begin{aligned}
& \left|K_{C}+\frac{1}{\delta^{2}} \sum_{j=1}^{2} \int_{t}^{u} d s \int_{\sigma_{s}}^{s}\left(s-\rho_{1}\right) \mathbb{E}\left[\mathbf{1}_{\mathfrak{X}^{c}\left(k-1, p_{4}\right)}\left(\boldsymbol{y}^{(\delta)}\left(\sigma_{s}\right)\right) \partial_{j} G\left(\boldsymbol{l}^{(\delta)}\left(\sigma_{s}\right)\right) \Lambda\left(\sigma_{s}, P\right) V_{j}\left(P_{\delta}\right) \hat{\zeta}\right] d \rho_{1}\right| \\
& \leq \frac{C}{\delta^{2}}\|G\|_{1} \mathbb{E}[\hat{\zeta}] \int_{G_{J}} d s \int_{\sigma_{s}}^{s}\left(s-\rho_{1}\right) \phi^{1 / 2}\left(C \frac{s-\rho_{1}}{\delta}\right) \phi^{1 / 2}\left(C \frac{\rho_{1}-\sigma_{s}}{\delta}\right) d \rho_{1}+C \delta^{1 / 6}(u-t)\|G\|_{1} \mathbb{E}[\hat{\zeta}] \\
& \leq \frac{C}{\delta^{2}}\|G\|_{1} \mathbb{E}[\hat{\zeta}] \int_{t}^{u} d s \int_{\sigma_{s}}^{s}\left(s-\rho_{1}\right) \phi^{1 / 2}\left(C \frac{s-\rho_{1}}{\delta}\right) \phi^{1 / 2}\left(C \frac{\rho_{1}-\sigma_{s}}{\delta}\right) d \rho_{1}+C \delta^{1 / 6}(u-t)\|G\|_{1} \mathbb{E}[\hat{\zeta}] \\
& \leq C \delta^{1 / 6}(u-t)\|G\|_{1} \mathbb{E} \hat{\zeta} .
\end{aligned}
$$


Next, we simplify the second term in the left-most part of (4.30). Using the fact that

$$
\left|\boldsymbol{l}^{(\delta)}(\rho)-\boldsymbol{l}^{(\delta)}\left(\sigma_{s}\right)\right| \leq C \delta^{1 / 2-\gamma_{A}}, \quad \rho \in\left[\sigma_{s}, s\right]
$$

as well as the estimate (4.5) and Lemma 3.1 we can argue that

$$
\left|\Lambda\left(\sigma_{s}, P\right)-\bar{\Theta}^{2}(s)\right| \leq C\left[p_{2}\left(N_{1} N_{2} N_{3} N_{4}\right)^{1 / 2} \delta^{1 / 2-\gamma_{A}}+p_{2}^{2} \delta^{3 / 2-2 \gamma_{A}}\right] \leq C \delta^{1 / 6}
$$

under our assumptions on $\epsilon_{j}$. and $\gamma_{A}$. We conclude therefore that the magnitude of the difference between the second term on the left hand side of (4.30) and

$$
\frac{1}{\delta^{2}} \sum_{j=1}^{2} \int_{t}^{u} \mathbb{E}\left[\partial_{j} G\left(\boldsymbol{l}^{(\delta)}\left(\sigma_{s}\right)\right) \bar{\Theta}^{2}(s)\left(\int_{\sigma_{s}}^{s}\left(s-\rho_{1}\right) V_{j}\left(P_{\delta}\right) d \rho_{1}\right) \hat{\zeta}\right] d s
$$

can be estimated by $C \delta^{1 / 6}(u-t)\|G\|_{1} \mathbb{E}[\hat{\zeta}]$. For $s \geq t+\delta^{1-\gamma_{A}}$ we can write the integral from $\sigma_{s}$ to $s$ appearing above as

$$
\frac{1}{\delta^{2}} \int_{s-\delta^{1-\gamma_{A}}}^{s}\left(s-\rho_{1}\right) \Delta R_{j}\left(\frac{s-\rho_{1}}{\delta} l^{(\delta)}\left(\sigma_{s}\right)\right) d \rho_{1}
$$

which upon the change of variables $\rho_{1}:=\left(s-\rho_{1}\right) / \delta$ is equal to

$$
\int_{0}^{\delta^{-\gamma}} \rho_{1} \Delta R_{j}\left(\rho_{1} \boldsymbol{l}^{(\delta)}\left(\sigma_{s}\right)\right) d \rho_{1}
$$

Since the expression corresponding to the integral over $s \in\left[t, t+\delta^{1-\gamma_{A}}\right]$ can be estimated by $C \delta^{1-3 \gamma_{A}}$ we conclude that

$$
\begin{aligned}
& \left|K_{C}+\sum_{j=1}^{2} \int_{t}^{u} \mathbb{E}\left[\mathbf{1}_{\mathfrak{X}^{c}\left(k-1, p, p_{4}\right)}\left(\boldsymbol{y}^{(\delta)}\left(\sigma_{s}\right)\right) \bar{\Theta}^{2}(s) \partial_{j} G\left(\boldsymbol{l}^{(\delta)}\left(\sigma_{s}\right)\right) \int_{0}^{\delta^{-\gamma_{A}}} \rho_{1} \Delta R_{j}\left(\rho_{1} \boldsymbol{l}^{(\delta)}\left(\sigma_{s}\right)\right) d \rho_{1} \hat{\zeta}\right] d s\right| \\
& \leq C \delta^{1 / 6}(u-t)\|G\|_{1} \mathbb{E} \hat{\zeta} .
\end{aligned}
$$

Thanks to (2.3) we can replace the integral from 0 to $\delta^{-\gamma_{A}}$ appearing on the left hand side of (4.33) by the improper integral from 0 to $+\infty$. Finally, it is straightforward to check that under the assumptions on $\epsilon_{i}$ in Proposition 3.5 and with $\gamma_{A}$ as in (4.4) all the conditions on $\epsilon_{i}$ that we have encountered in this section are satisfied. We obtain, therefore,

$$
\left|\mathbb{E}\left\{\left[I^{(1)}-\sum_{j=1}^{2} \int_{t}^{u} E_{j}\left(\boldsymbol{l}^{(\delta)}\left(\sigma_{s}\right)\right) \bar{\Theta}^{2}(s) \partial_{j} G\left(\boldsymbol{l}^{(\delta)}\left(\sigma_{s}\right)\right) d s\right] \hat{\zeta}\right\}\right| \leq C \delta^{1 / 6}\left[(u-t) \vee \frac{1}{p_{3}}\right]\|G\|_{1} \mathbb{E} \hat{\zeta}
$$

for a certain constant $C>0$ and $E_{j}(\cdot)$ given by (2.10). Hence, (4.7) has been verified - it remains only to observe that the argument $\sigma_{s}$ in (4.34) can be replaced by $s$ making a small error using the regularity of the field $H(\mathbf{x})$ and estimate (4.31). 


\subsection{The proof of (4.8) and (4.9)}

The calculations concerning these terms essentially follow the respective steps performed in the previous section so we only highlight their main points. First, using Lemma 3.1] and (4.31) we note that the difference between $\mathbb{E}\left[I^{(2)} \hat{\zeta}\right]$ and

$$
\frac{1}{\delta} \sum_{i, j=1}^{2} \int_{t}^{u} d s \int_{\sigma_{s}}^{s} \mathbb{E}\left[\partial_{j} G\left(\boldsymbol{l}^{(\delta)}\left(\sigma_{s}\right)\right) \partial_{\ell_{i}} F_{j, \delta}\left(s, \frac{\boldsymbol{y}^{(\delta)}(s)}{\delta}, \boldsymbol{l}^{(\delta)}\left(\sigma_{s}\right)\right) F_{i, \delta}\left(\rho, \frac{\boldsymbol{y}^{(\delta)}(\rho)}{\delta}, \boldsymbol{l}^{(\delta)}\left(\sigma_{s}\right)\right) \hat{\zeta}\right] d \rho
$$

is less than, or equal to

$$
C \delta^{1 / 2-2 \gamma_{A}} p_{2}\left(N_{1} N_{2} N_{3} N_{4}\right)^{1 / 2}(u-t)\|G\|_{2} \mathbb{E} \hat{\zeta} \leq C \delta^{1 / 6}(u-t)\|G\|_{1} \mathbb{E} \hat{\zeta}
$$

under our assumptions on $\epsilon_{i}$ and $\gamma_{A}$. Next, we note that (4.35) equals to

$$
\begin{gathered}
\frac{1}{\delta} \sum_{i, j=1}^{2} \int_{t}^{u} d s \int_{\sigma_{s}}^{s} \mathbb{E}\left[\partial_{j} G\left(\boldsymbol{l}^{(\delta)}\left(\sigma_{s}\right)\right) \partial_{\ell_{i}} F_{j, \delta}\left(s, \frac{\boldsymbol{L}^{(\delta)}\left(\sigma_{s}, s\right)}{\delta}, \boldsymbol{l}^{(\delta)}\left(\sigma_{s}\right)\right) F_{i, \delta}\left(\rho, \frac{\boldsymbol{L}^{(\delta)}\left(\sigma_{s}, \rho\right)}{\delta}, \boldsymbol{l}^{(\delta)}\left(\sigma_{s}\right)\right) \hat{\zeta}\right] d \rho \\
+\frac{1}{\delta^{2}} \sum_{i, j, k=1}^{2} \int_{t}^{u} d s \int_{\sigma_{s}}^{s} \int_{0}^{1} \mathbb{E}\left[\partial_{j} G\left(\boldsymbol{l}^{(\delta)}\left(\sigma_{s}\right)\right) \partial_{\ell_{i}} \partial_{y_{k}} F_{j, \delta}\left(s, \frac{\boldsymbol{R}^{(\delta)}\left(v, \sigma_{s}, s\right)}{\delta}, \boldsymbol{l}^{(\delta)}\left(\sigma_{s}\right)\right)\right. \\
\left.\quad \times F_{i, \delta}\left(\rho, \frac{\boldsymbol{L}^{(\delta)}\left(\sigma_{s}, \rho\right)}{\delta}, \boldsymbol{l}^{(\delta)}\left(\sigma_{s}\right)\right)\left(y_{k}^{(\delta)}(s)-L_{k}^{(\delta)}\left(\sigma_{s}, s\right)\right) \hat{\zeta}\right] d \rho d v \\
+\frac{1}{\delta^{2}} \sum_{i, j, k=1}^{2} \int_{t}^{u} d s \int_{\sigma_{s}}^{s} \int_{0}^{1} \mathbb{E}\left[\partial_{j} G\left(\boldsymbol{l}^{(\delta)}\left(\sigma_{s}\right)\right) \partial_{\ell_{i}} F_{j, \delta}\left(s, \frac{\boldsymbol{y}^{(\delta)}(s)}{\delta}, \boldsymbol{l}^{(\delta)}\left(\sigma_{s}\right)\right)\right. \\
\left.\times \partial_{y_{k}} F_{i, \delta}\left(\rho, \frac{\boldsymbol{R}^{(\delta)}\left(v, \sigma_{s}, \rho\right)}{\delta}, \boldsymbol{l}^{(\delta)}\left(\sigma_{s}\right)\right)\left(y_{k}^{(\delta)}(\rho)-L_{k}^{(\delta)}\left(\sigma_{s}, \rho\right)\right) \hat{\zeta}\right] d \rho d v .
\end{gathered}
$$

A straightforward argument using Lemma 3.1] and (4.5) shows that both the second and third terms of (4.36) can be estimated by

$$
C \delta^{1 / 2-3 \gamma_{A}} p_{2}\left(N_{1} N_{2} N_{3} N_{4}\right)^{1 / 2}(u-t)\|G\|_{1} \mathbb{E} \hat{\zeta} \leq C \delta^{1 / 6}(u-t)\|G\|_{1} \mathbb{E} \hat{\zeta} .
$$

The first term, on the other hand, can be handled with the help of part (ii) of Lemma 3.4 in the same fashion as we have dealt with the term $J_{1}^{(2)}$, given by (4.22) of Section 4.1] in the process we have to use Lemma 3.1 in order to estimate $\partial_{\ell_{i}} F_{j, \delta}$. As a result we obtain that

$$
\begin{aligned}
& \left|\mathbb{E}\left\{\left[I^{(2)}-\sum_{j=1}^{2} \int_{t}^{u} \tilde{J}_{j}\left(s ; \boldsymbol{y}^{(\delta)}(\cdot), \boldsymbol{l}^{(\delta)}(\cdot)\right) \bar{\Theta}(s) \partial_{j} G\left(\boldsymbol{l}^{(\delta)}\left(\sigma_{s}\right)\right) d s\right] \hat{\zeta}\right\}\right| \\
& \leq \frac{C}{\delta} \frac{p_{2}}{p_{1}} \delta^{2-2 \gamma_{A}} p_{2}\left(N_{1} N_{2} N_{3} N_{4}\right)^{1 / 2} p_{3}\left[(u-t) \vee \frac{1}{p_{3}}\right]\|G\|_{1} \mathbb{E} \hat{\zeta} \leq C \delta^{1 / 6}\left[(u-t) \vee \frac{1}{p_{3}}\right]\|G\|_{1} \mathbb{E} \hat{\zeta}
\end{aligned}
$$

with

$$
\tilde{J}_{j}\left(s ; \boldsymbol{y}^{(\delta)}(\cdot), \boldsymbol{l}^{(\delta)}(\cdot)\right):=-\sum_{i=1}^{d} \bar{\Theta}_{i}(s) D_{i, j}\left(\boldsymbol{l}^{(\delta)}\left(\sigma_{s}\right)\right),
$$




$$
\begin{gathered}
\bar{\Theta}_{i}(s):=\partial_{l_{i}} \Theta\left(s, \boldsymbol{y}^{(\delta)}(s), \boldsymbol{l}^{(\delta)}(s) ; \boldsymbol{y}^{(\delta)}(\cdot), \boldsymbol{l}^{(\delta)}(\cdot)\right) \\
I^{(3)}:=\frac{1}{\delta} \sum_{i, j=1}^{2} \int_{t}^{u} d s \int_{\sigma_{s}}^{s} \partial_{i, j}^{2} G\left(\boldsymbol{l}^{(\delta)}(\rho)\right) F_{j, \delta}\left(s, \frac{\boldsymbol{y}^{(\delta)}(s)}{\delta}, \boldsymbol{l}^{(\delta)}(\rho)\right) F_{i, \delta}\left(\rho, \frac{\boldsymbol{y}^{(\delta)}(\rho)}{\delta}, \boldsymbol{l}^{(\delta)}(\rho)\right) d \rho
\end{gathered}
$$

Finally, concerning the limit of $\mathbb{E}\left[I^{(3)} \hat{\zeta}\right]$, we use Lemma 3.1] 4.5], and t4.31) to conclude that

$$
\begin{aligned}
& \left|\mathbb{E}\left[I^{(3)} \hat{\zeta}\right]-\mathcal{I}\right| \leq \frac{C}{\delta}\left[p_{2}\left(N_{1} N_{2} N_{3} N_{4}\right)^{1 / 2} \delta^{1 / 2-\gamma_{A}}+p_{2}^{2} \delta^{1 / 2-2 \gamma_{A}}\right] \delta^{1-\gamma_{A}}(u-t)\|G\|_{1} \mathbb{E} \hat{\zeta} \\
& \leq C \delta^{1 / 6}(u-t)\|G\|_{1} \mathbb{E} \hat{\zeta}
\end{aligned}
$$

where

$$
\mathcal{I}:=\frac{1}{\delta} \int_{t}^{u} \int_{\sigma_{s}}^{s} \mathbb{E}\left\{\partial_{i, j}^{2} G\left(\boldsymbol{l}^{(\delta)}\left(\sigma_{s}\right)\right) F_{j, \delta}\left(s, \frac{\boldsymbol{L}^{(\delta)}\left(\sigma_{s}, s\right)}{\delta}, \boldsymbol{l}^{(\delta)}\left(\sigma_{s}\right)\right) F_{i, \delta}\left(\rho, \frac{\boldsymbol{L}^{(\delta)}\left(\sigma_{s}, \rho\right)}{\delta}, \boldsymbol{l}^{(\delta)}\left(\sigma_{s}\right)\right) \hat{\zeta}\right\} d s d \rho .
$$

Then, we can use part (ii) of Lemma 3.4 and obtain

$$
\left|\mathcal{I}-\sum_{i, j=1}^{2} \int_{t}^{u} D_{i, j}\left(\boldsymbol{l}^{(\delta)}\left(\sigma_{s}\right)\right) \bar{\Theta}^{2}(s) \partial_{i, j}^{2} G\left(\boldsymbol{l}^{(\delta)}\left(\sigma_{s}\right)\right) d s\right| \leq C \delta^{1 / 6}\left[(u-t) \vee \frac{1}{p_{3}}\right]\|G\|_{2} \mathbb{E} \hat{\zeta} .
$$

Finally, we replace the argument $\sigma_{s}$, in formulas (4.34), (4.37) and (4.38), by $s$. This can be done thanks to estimate (4.31) and the assumption on the regularity of the random field $H(\cdot)$. We remark that in order to make this approximation work we will be forced to use the third derivative of $G(\cdot)$. This finishes the proof of Lemma 4.1 and Proposition 3.5 ,

\section{The proof of Theorem 2.1}

\subsection{An estimate of $\tilde{Q}_{\mathbf{x}, \mathbf{v}}^{(\delta)}\left[\tau_{\delta} \leq T\right]$}

The principal result of this section is the following estimate on the stopping time for the process with cut-offs.

Theorem 5.1 There exist $C, \gamma>0$ such that

$$
\tilde{Q}_{\mathbf{x}, \mathbf{v}}^{(\delta)}\left[\tau_{\delta} \leq T\right] \leq C \delta^{\gamma}
$$

We start with the following construction of the augmentation of path measures that has been carried out in Section 6.1 of [8]. Let $s \geq 0$ be fixed and $\pi \in \mathcal{C}$. Then, according to Lemma 6.1.1 of ibid., there exists a unique probability measure, that is denoted by $\delta_{\pi} \otimes_{s} \mathfrak{Q}_{X(s), V(s)}$, such that for any pair of events $A \in \mathcal{M}^{s}, B \in \mathcal{M}$ we have $\delta_{\pi} \otimes_{s} \mathfrak{Q}_{X(s), V(s)}[A]=\mathbf{1}_{A}(\pi)$ and $\delta_{\pi} \otimes_{s} \mathfrak{Q}_{X(s), V(s)}\left[\theta_{s}(B)\right]=$ $\mathfrak{Q}_{X(s), V(s)}[B]$. The following result is a direct consequence of Theorem 6.2 .1 of [8].

Proposition 5.2 There exists a unique probability measure $R_{\mathbf{x}, \mathbf{v}}^{(\delta)}$ on $\mathcal{C}$ such that

$$
R_{\mathbf{x}, \mathbf{v}}^{(\delta)}[A]:=\tilde{Q}_{\mathbf{x}, \mathbf{v}}^{(\delta)}[A]
$$

for all $A \in \mathcal{M}^{\tau_{\delta}}$ and the regular conditional probability distribution of $R_{\mathbf{x}, \mathbf{v}}^{(\delta)}\left[\cdot \mid \mathcal{M}^{\tau_{\delta}}\right]$ is given by $\delta_{\pi} \otimes_{\tau_{\delta}(\pi)} \mathfrak{Q}_{X\left(\tau_{\delta}(\pi)\right), V\left(\tau_{\delta}(\pi)\right)}, \pi \in \mathcal{C}$. This measure shall be also denoted by $\tilde{Q}_{\mathbf{x}, \mathbf{v}}^{(\delta)} \otimes_{\tau_{\delta}} \mathfrak{Q}_{X\left(\tau_{\delta}\right), V\left(\tau_{\delta}\right)}$. 
We denote by $E_{\mathbf{x}, \mathbf{v}}^{(\delta)}$ the expectation with respect to the augmented measure $R_{\mathbf{x}, \mathbf{v}}^{(\delta)}$. In particular (5.2) proves that

$$
R_{\mathbf{x}, \mathbf{v}}^{(\delta)}\left[\tau_{\delta} \leq T\right]=\tilde{Q}_{\mathbf{x}, \mathbf{v}}^{(\delta)}\left[\tau_{\delta} \leq T\right]
$$

We obviously have

$$
\left[\tau_{\delta} \leq T\right]=\left[U_{\delta} \leq \tau_{\delta}, U_{\delta} \leq T\right] \cup\left[S_{\delta} \leq \tau_{\delta}, S_{\delta} \leq T\right]
$$

Here $U_{\delta}$, see (3.5), is the stopping time associated with the nearly tangential passing of the past trajectory and $S_{\delta}$, see (3.6), is the stopping time corresponding to violent turns on either of the scales $1 / p_{i}, i=1,2,3$. Let us denote the first and second event appearing on the right hand side of (5.4) by $A(\delta)$ and $B(\delta)$ respectively:

$$
A(\delta)=\left[U_{\delta} \leq \tau_{\delta}, U_{\delta} \leq T\right], \quad B(\delta)=\left[S_{\delta} \leq \tau_{\delta}, S_{\delta} \leq T\right] .
$$

These events are $\mathcal{M}^{\tau_{\delta}}$-measurable. Hence

$$
R_{\mathbf{x}, \mathbf{v}}^{(\delta)}[A(\delta)]=\tilde{Q}_{\mathbf{x}, \mathbf{v}}^{(\delta)}[A(\delta)] \quad \text { and } \quad R_{\mathbf{x}, \mathbf{v}}^{(\delta)}[B(\delta)]=\tilde{Q}_{\mathbf{x}, \mathbf{v}}^{(\delta)}[B(\delta)] .
$$

We will estimate the $\tilde{Q}_{\mathbf{x}, \mathbf{v}}^{(\delta)}$ probabilities of these two events separately and will show that both can be estimated by $C \delta^{\gamma}$ for certain constants $C, \gamma>0$.

\subsection{An estimate of $\tilde{Q}_{\mathrm{x}, \mathrm{v}}^{(\delta)}[A(\delta)]$.}

According to the remarks form the previous section it suffices only to show that there exist $C, \gamma>0$ such that

$$
R_{\mathbf{x}, \mathbf{v}}^{(\delta)}[A(\delta)] \leq C \delta^{\gamma} .
$$

The following proposition is a consequence of Proposition 3.5 and the construction of the augmented measure. To abbreviate the notation we let

$$
\left.N_{t}(G):=G(t, X(t), V(t))-G(0, X(0), V(0))-\int_{0}^{t}\left(\partial_{\varrho}+\tilde{\mathcal{L}}\right) G(\varrho, X(\varrho), V(\varrho))\right) d \varrho
$$

for any $G \in C_{b}^{1,1,3}\left([0,+\infty) \times \mathbb{R}_{*}^{4}\right)$ and $t \geq 0$. Here $\tilde{\mathcal{L}}$ is the generator of the degenerate diffusion given by (2.11).

Proposition 5.3 Suppose that $(\mathbf{x}, \mathbf{v}) \in \mathcal{A}(M)$ and $\zeta \in C_{b}\left(\left(\mathbb{R}_{*}^{4}\right)^{n}\right)$ is nonnegative. Let $0 \leq t_{1}<$ $\ldots<t_{n} \leq t<u \leq T$. Then, there exists a constant $C>0$ such that for any function $G \in$ $C_{b}^{1,1,3}\left([0,+\infty) \times \mathbb{R}_{*}^{4}\right)$ we have

$$
\left|E_{\mathbf{x}, \mathbf{v}}^{(\delta)}\left\{\left[N_{u}(G)-N_{t}(G)\right] \tilde{\zeta}\right\}\right| \leq C \delta^{1 / 30}\|G\|_{1,1,3} E_{\mathbf{x}, \mathbf{v}}^{(\delta)} \tilde{\zeta}
$$

Here $\tilde{\zeta}:=\zeta\left(X\left(t_{1}\right), V\left(t_{1}\right), \ldots, X\left(t_{n}\right), V\left(t_{n}\right)\right)$. The choice of $C>0$ does not depend on $(\mathbf{x}, \mathbf{v}), \delta \in$ $(0,1], \zeta$, times $t_{1}, \ldots, t_{n}, u, t$, or the function $G$.

Proof. Let $0=s_{0} \leq s_{1} \leq \ldots \leq s_{n} \leq t$ and $B_{1}, \ldots, B_{n} \in \mathcal{B}\left(\mathbb{R}_{*}^{4}\right)$ be Borel sets. We denote $A_{0}:=\mathcal{C}$ and for any $k \in\{1, \ldots, n\}, s \leq s_{k}$ we define the events

$$
A_{k}:=\left[\pi:\left(X\left(s_{1}\right), V\left(s_{1}\right)\right) \in B_{1}, \ldots,\left(X\left(s_{k}\right), V\left(s_{k}\right)\right) \in B_{k}\right]
$$

and their shifted counterparts

$$
A_{k}^{(s)}:=\left[\pi:\left(X\left(s_{k}-s\right), V\left(s_{k}-s\right)\right) \in B_{k}, \ldots,\left(X\left(s_{n}-s\right), V\left(s_{n}-s\right)\right) \in B_{n}\right] .
$$


We write

$$
\begin{aligned}
E_{\mathbf{x}, \mathbf{v}, \pi}^{(\delta)}\left[N_{u}(G)-N_{u \wedge \tau_{\delta}(\pi)}(G), A_{n}\right]= & \sum_{p=0}^{n-1} \mathbf{1}_{\left[s_{p}, s_{p+1}\right)}\left(\tau_{\delta}(\pi)\right) \mathbf{1}_{A_{p}}(\pi) \mathfrak{M}_{X\left(\tau_{\delta}(\pi)\right), V\left(\tau_{\delta}(\pi)\right)}\left[N_{u-\tau_{\delta}(\pi)}(G), A_{p+1}^{\left(\tau_{\delta}(\pi)\right)}\right] \\
& +\mathbf{1}_{\left[s_{n}, u\right)}\left(\tau_{\delta}(\pi)\right) \mathbf{1}_{A_{n}}(\pi) \mathfrak{M}_{X\left(\tau_{\delta}(\pi)\right), V\left(\tau_{\delta}(\pi)\right)}\left[N_{u-\tau_{\delta}(\pi)}(G)\right] .
\end{aligned}
$$

When $\tau_{\delta}(\pi) \in\left[s_{p}, s_{p+1}\right)$ we obviously have

$$
\mathfrak{M}_{X\left(\tau_{\delta}(\pi)\right), V\left(\tau_{\delta}(\pi)\right)}\left[N_{u-\tau_{\delta}(\pi)}(G), A_{p+1}^{\left(\tau_{\delta}(\pi)\right)}\right]=\mathfrak{M}_{X\left(\tau_{\delta}(\pi)\right), V\left(\tau_{\delta}(\pi)\right)}\left[N_{t-\tau_{\delta}(\pi)}(G), A_{p+1}^{\left(\tau_{\delta}(\pi)\right)}\right]
$$

and $\mathfrak{M}_{X\left(\tau_{\delta}(\pi)\right), V\left(\tau_{\delta}(\pi)\right)} N_{u-\tau_{\delta}(\pi)}(G)=0$. Hence the left hand side of (5.10) equals

$$
\begin{aligned}
& \sum_{p=0}^{n-1} \mathbf{1}_{\left[s_{p}, s_{p+1}\right)}\left(\tau_{\delta}(\pi)\right) \mathbf{1}_{A_{p}}(\pi) \mathfrak{M}_{X\left(\tau_{\delta}(\pi)\right), V\left(\tau_{\delta}(\pi)\right)}\left[N_{t-\tau_{\delta}(\pi)}(G), A_{p+1}^{\left(\tau_{\delta}(\pi)\right)}\right] \\
& =E_{\mathbf{x}, \mathbf{v}, \pi}^{(\delta)}\left[N_{t}(G)-N_{t \wedge \tau_{\delta}(\pi)}(G), A_{n}\right] .
\end{aligned}
$$

We conclude from (5.10), (5.11) that

$$
\begin{aligned}
E_{\mathbf{x}, \mathbf{v}, \pi}^{(\delta)}\left[N_{u}(G), A_{n}\right] & =E_{\mathbf{x}, \mathbf{v}, \pi}^{(\delta)}\left[N_{u \wedge \tau_{\delta}(\pi)}(G)+N_{t}(G)-N_{t \wedge \tau_{\delta}(\pi)}(G), A_{n}\right] \\
& =E_{\mathbf{x}, \mathbf{v}, \pi}^{(\delta)}\left[N_{\left(u \wedge \tau_{\delta}(\pi)\right) \vee t}(G), A_{n}\right]
\end{aligned}
$$

and therefore

$$
\begin{aligned}
& E_{\mathbf{x}, \mathbf{v}}^{(\delta)}\left[N_{u}(G), A_{n}\right]=E_{\mathbf{x}, \mathbf{v}}^{(\delta)}\left[E_{\mathbf{x}, \mathbf{v}, \pi}^{(\delta)}\left[N_{\left(u \wedge \tau_{\delta}(\pi)\right) \vee t}(G), A_{n}\right]\right] \\
& =E_{\mathbf{x}, \mathbf{v}}^{(\delta)}\left[E_{\mathbf{x}, \mathbf{v}, \pi}^{(\delta)}\left[N_{\left(u \wedge \tau_{\delta}(\pi)\right) \vee t}(G), A_{n}\right], \tau_{\delta}(\pi) \leq t\right]+E_{\mathbf{x}, \mathbf{v}}^{(\delta)}\left[E_{\mathbf{x}, \mathbf{v}, \pi}^{(\delta)}\left[N_{\left(u \wedge \tau_{\delta}(\pi)\right) \vee t}(G), A_{n}\right], \tau_{\delta}(\pi)>t\right] .
\end{aligned}
$$

Let $B:=A_{n} \cap\left[\tau_{\delta}>t\right]$. Note that it is an $\mathcal{M}^{t}$-measurable event. The first term on the utmost right hand side of (5.13) equals

$$
E_{\mathbf{x}, \mathbf{v}}^{(\delta)}\left[E_{\mathbf{x}, \mathbf{v}, \pi}^{(\delta)}\left[N_{t}(G), A_{n}\right], \tau_{\delta}(\pi) \leq t\right]=E_{\mathbf{x}, \mathbf{v}}^{(\delta)}\left[N_{t}(G), A_{n}\right]-\tilde{E}_{\mathbf{x}, \mathbf{v}}^{(\delta)}\left[N_{t}(G), B\right],
$$

while the second one equals $\tilde{E}_{\mathbf{x}, \mathbf{v}}^{(\delta)}\left[N_{\left(u \wedge \tau_{\delta}\right) \vee t}(G), B\right]$. It follows that

$$
E_{\mathbf{x}, \mathbf{v}}^{(\delta)}\left[N_{u}(G)-N_{t}(G), A_{n}\right]=\tilde{E}_{\mathbf{x}, \mathbf{v}}^{(\delta)}\left[N_{\left(u \wedge \tau_{\delta}\right) \vee t}(G), B\right]-\tilde{E}_{\mathbf{x}, \mathbf{v}}^{(\delta)}\left[N_{t}(G), B\right] .
$$

We define

$$
\sigma:=p_{3}^{-1}\left[\left(\left[p_{3}\left(u \wedge \tau_{\delta}\right)\right]+1\right) \vee\left(\left[p_{3} t\right]+1\right)\right]
$$

as a point on the $1 / p_{3}$-mesh that approximates $\left(u \wedge \tau_{\delta}\right) \vee t$, and note that

$$
\tilde{E}_{\mathbf{x}, \mathbf{v}}^{(\delta)}\left[N_{\sigma}(G), B\right]=\sum_{r=\left[p_{3} t\right]+1}^{\left[p_{3} u\right]+1} \tilde{E}_{\mathbf{x}, \mathbf{v}}^{(\delta)}\left[N_{r / p_{3}}(G), B, \sigma=\frac{r}{p_{3}}\right] .
$$

Representing the event $\left[\sigma=r / p_{3}\right]$ as the difference of $\left[\sigma \geq r / p_{3}\right]$ and $\left[\sigma \geq(r+1) / p_{3}\right]$ (note that $\left.\left[\sigma \geq\left(\left[p_{3} u\right]+1\right) / p_{3}\right]=\emptyset\right)$ and grouping the terms of the sum that correspond to the same index $r$ we obtain that the right hand side of (5.15) equals

$$
\tilde{E}_{\mathbf{x}, \mathbf{v}}^{(\delta)}\left[N_{\left(\left[p_{3} t\right]+1\right) / p_{3}}(G), B\right]+\sum_{r=\left[p_{3} t\right]+1}^{\left[p_{3} u\right]+1} \tilde{E}_{\mathbf{x}, \mathbf{v}}^{(\delta)}\left[N_{(r+1) / p_{3}}(G)-N_{r / p_{3}}(G), B, \sigma \geq \frac{r+1}{p_{3}}\right] .
$$


Since the event $B \cap\left[\sigma \geq(r+1) / p_{3}\right]$ is $\mathcal{M}^{r / p_{3}}$-measurable, from Proposition 3.5] we conclude that the absolute value of each term appearing under the summation sign in (5.16) can be estimated by $C\|G\|_{1,1,3} \delta^{1 / 6} p_{3}^{-1} \tilde{Q}_{\mathbf{x}, \mathbf{v}}^{(\delta)}[B]$ which implies

$$
\left|\tilde{E}_{\mathbf{x}, \mathbf{v}}^{(\delta)}\left[N_{\sigma}(G), B\right]-\tilde{E}_{\mathbf{x}, \mathbf{v}}^{(\delta)}\left[N_{\left(\left[p_{3} t\right]+1\right) / p_{3}}(G), B\right]\right| \leq C \delta^{1 / 6}\|G\|_{1,1,3} \tilde{Q}_{\mathbf{x}, \mathbf{v}}^{(\delta)}[B] \frac{\left[p_{3} u\right]+1-\left[p_{3} t\right]}{p_{3}} .
$$

Next, using (A.2) we obtain that

$$
\left|V(\sigma)-V\left(\left(u \wedge \tau_{\delta}\right) \vee t\right)\right| \leq C N_{3}^{-1 / 2}
$$

and

$$
\left|V\left(\left(\left[p_{3} t\right]+1\right) p_{3}^{-1}\right)-V(t)\right| \leq C N_{3}^{-1 / 2},
$$

$\tilde{Q}_{\mathbf{x}, \mathbf{v}}^{(\delta)}$-a.s. We note that this is the only place in the proof of Theorem 2.1 where the "no violent turn on the scale $1 / p_{3}$ " stopping time $S_{\delta}^{(3)}$ is used. From the definition of the cut-off dynamics, see (3.17), we also have

$$
\left|X(\sigma)-X\left(\left(u \wedge \tau_{\delta}\right) \vee t\right)\right| \leq M_{*} p_{3}^{-1}
$$

and

$$
\left|X\left(\left(\left[p_{3} t\right]+1\right) p_{3}^{-1}\right)-X(t)\right| \leq M_{*} p_{3}^{-1} .
$$

As a consequence, both $\left|N_{\sigma}(G)-N_{\left(u \wedge \tau_{\delta}\right) \vee t}(G)\right|$ and $\left|N_{\left(\left[p_{3} t\right]+1\right) p_{3}^{-1}}(G)-N_{t}(G)\right|$ may be estimated by $C\|G\|_{1,1,3} N_{3}^{-1 / 2}$, as $N_{3} \ll p_{3}$. Since, as we recall, $N_{3}=\left[\delta^{-\epsilon / 7}\right]$, where $\epsilon_{7} \in(1 / 15,1 / 10)$, we have

$$
\begin{aligned}
& \left|\tilde{E}_{\mathbf{x}, \mathbf{v}}^{(\delta)}\left[N_{\left(u \wedge \tau_{\delta}\right) \vee t}(G), B\right]-\tilde{E}_{\mathbf{x}, \mathbf{v}}^{(\delta)}\left[N_{t}(G), B\right]\right| \leq\left|\tilde{E}_{\mathbf{x}, \mathbf{v}}^{(\delta)}\left[N_{\sigma}(G)-N_{\left(u \wedge \tau_{\delta}\right) \vee t}(G), B\right]\right| \\
& +\left|\tilde{E}_{\mathbf{x}, \mathbf{v}}^{(\delta)}\left[N_{\sigma}(G), B\right]-\tilde{E}_{\mathbf{x}, \mathbf{v}}^{(\delta)}\left[N_{\left(\left[p_{3} t\right]+1\right) p_{3}^{-1}}(G), B\right]\right|+\left|\tilde{E}_{\mathbf{x}, \mathbf{v}}^{(\delta)}\left[N_{\left(\left[p_{3} t\right]+1\right) p_{3}^{-1}}(G)-N_{t}, B\right]\right| \\
& \leq C \delta^{1 / 30}\|G\|_{1,1,3} \tilde{Q}_{\mathbf{x}, \mathbf{v}}^{(\delta)}[B]
\end{aligned}
$$

for a certain constant $C>0$. From (5.14) and (5.17) we obtain

$$
\left|E_{\mathbf{x}, \mathbf{v}}^{(\delta)}\left[N_{u}(G)-N_{t}(G), A_{n}\right]\right| \leq C \delta^{1 / 30}\|G\|_{1,1,3} \tilde{Q}_{\mathbf{x}, \mathbf{v}}^{(\delta)}[B] \leq C \delta^{1 / 30}\|G\|_{1,1,3} \tilde{Q}_{\mathbf{x}, \mathbf{v}}^{(\delta)}\left[A_{n}\right]
$$

for a certain constant $C>0$ and the conclusion of Proposition 5.3 follows.

To simplify our notation we assume in the subsequent notation that $M_{*}=1$. Note that for $\delta \in\left(0, \delta_{*}\right]$, where $\delta_{*}$ is sufficiently small we have, using (3.21)

$A(\delta) \subset \tilde{A}(\delta):=\bigcup_{i, j=1}^{\left[T p_{2}\right]}\left[\left|\boldsymbol{y}^{(\delta)}\left(\frac{j}{p_{2}}\right)-\boldsymbol{y}^{(\delta)}\left(\frac{i}{p_{2}}\right)\right| \leq \frac{5}{p_{2}},\left|\hat{\boldsymbol{l}}^{(\delta)}\left(\frac{j}{p_{2}}\right) \cdot \hat{\boldsymbol{l}}^{(\delta)}\left(\frac{i}{p_{2}}\right)\right| \geq 1-\frac{8}{N_{4}}, j-i \geq \frac{p_{2}}{p_{1}}\right]$

and thus

$$
\begin{aligned}
& R_{\mathbf{x}, \mathbf{v}}^{(\delta)}[A(\delta)] \\
& \leq\left[T p_{2}\right]^{2} \max _{1 \leq i, j \leq\left[T p_{2}\right]}\left\{R_{\mathbf{x}, \mathbf{v}}^{(\delta)}\left[\left|\boldsymbol{y}^{(\delta)}\left(\frac{j}{p_{2}}\right)-\boldsymbol{y}^{(\delta)}\left(\frac{i}{p_{2}}\right)\right| \leq \frac{5}{p_{2}},\left|\hat{\boldsymbol{l}}^{(\delta)}\left(\frac{j}{p_{2}}\right) \cdot \hat{\boldsymbol{l}}^{(\delta)}\left(\frac{i}{p_{2}}\right)\right| \geq 1-\frac{8}{N_{4}}\right]\right\}
\end{aligned}
$$

with the maximum taken over $j-i \geq p_{2} / p_{1}$. In estimating the probability appearing on the right hand side of (5.19) we shall need the following. 
Lemma 5.4 Let $p_{1}, p_{2}$ be as in (3.1). Then, there exist positive constants $C_{1}, C_{2}$ and $C_{3}$ such that for all $\mathbf{x}, \mathbf{y} \in \mathbb{R}^{2},|\mathbf{w}|=|\mathbf{v}|=v, j \in\left\{1, \ldots,\left[p_{1} T\right]\right\}, \delta \in(0,1]$ we have

$$
\mathfrak{Q}_{\mathbf{x}, \mathbf{v}}\left[\left|X\left(\frac{j}{p_{1}}\right)-\mathbf{y}\right| \leq \frac{7}{p_{2}},\left|\hat{V}\left(\frac{j}{p_{1}}\right) \cdot \hat{\mathbf{w}}\right| \geq 1-\frac{9}{N_{4}}\right] \leq C_{1}\left(\frac{p_{1}^{C_{2}}}{p_{2}^{2} N_{4}^{1 / 2}}+e^{-C_{3} p_{1}}\right) .
$$

Here $\mathfrak{Q}_{\mathbf{x}, \mathbf{v}}$ is the path probability measure of the degenerate diffusion with the generator (2.8).

Proof. We prove this lemma by induction on $j$. First, we verify it for $j=1$. Without any loss of generality we may suppose that $\mathbf{v}=\left(v_{1}, v_{2}\right)$ and $v_{2}>1 / 8$. Let $\hat{D}: \mathbb{R} \rightarrow \mathbb{R}, \tilde{E}_{m}: \mathbb{R} \rightarrow \mathbb{R}, m=1,2$ be given by

$$
\hat{D}\left(v_{1}\right):=D_{11}\left(v_{1}, \sqrt{1-v_{1}^{2}}\right), \quad \tilde{E}_{m}\left(v_{1}\right):=E_{m}\left(v_{1}, \sqrt{1-v_{1}^{2}}\right),
$$

when $\mathbf{l} \in Z:=\left[v_{1}:\left|v_{1}\right| \leq(7 / 8)^{1 / 2}\right]$. These functions are $C^{\infty}$ smooth and bounded together with all their derivatives. It can be easily seen that $V_{1}(t), t \geq 0$, is a diffusion starting at $v_{1}$, whose generator $\mathcal{N}$ is of the form

$$
\mathcal{N} F\left(v_{1}\right):=\hat{D}^{1 / 2}\left(v_{1}\right) \partial_{v_{1}}\left(\hat{D}^{1 / 2}\left(v_{1}\right) \partial_{v_{1}} F\right)\left(v_{1}\right)+a\left(v_{1}\right) \partial_{v_{1}} F\left(v_{1}\right), \quad F \in C_{0}^{\infty}\left(\mathbb{R}_{*}\right),
$$

where $a(\cdot)$ is a certain $C^{\infty}$-function. Let

$$
\tilde{\mathcal{N}} F\left(v_{1}, \mathbf{x}\right):=\hat{D}^{1 / 2}\left(v_{1}\right) \partial_{v_{1}}\left(\hat{D}^{1 / 2}\left(v_{1}\right) \partial_{v_{1}} F\right)\left(v_{1}, \mathbf{x}\right)+\tilde{X}_{0} F\left(v_{1}, \mathbf{x}\right), \quad F \in C_{0}^{\infty}\left(\mathbb{R}_{*} \times \mathbb{R}^{2}\right),
$$

where $\tilde{X}_{0}$ is a $C^{\infty}$-smooth extension of the field

$$
X_{0}\left(v_{1}\right):=\left(a\left(v_{1}\right) \partial_{v_{1}}+v_{1} \partial_{x_{1}}+\sqrt{1-v_{1}^{2}} \partial_{x_{2}}\right), \quad v_{1} \in Z
$$

It can be shown, by the same type of argument as that given on pp. 122-123 of [1], that for each $\left(\mathbf{x}, v_{1}\right)$, with $v_{1} \in Z$, the linear space spanned at that point by the fields belonging to the Lie algebra generated by $\left[X_{0}, X_{1}\right], X_{1}$ is of dimension 3 . One can also ensure that the extensions $\left[\tilde{X}_{0}, \tilde{X}_{1}\right], \tilde{X}_{1}$ satisfy the same condition. We shall denote the respective extension of $\mathcal{N}$ by the same symbol. Let $\mathcal{R}_{v_{0}}, \tilde{\mathcal{R}}_{\mathbf{x}, v_{0}}$ be the probability measures supported on the respective path spaces $\mathcal{C}^{1}:=C([0,+\infty) ; \mathbb{R})$, $\mathcal{C}^{3}:=C\left([0,+\infty) ; \mathbb{R}^{3}\right)$ that solve the martingale problems corresponding to the generators $\mathcal{N}$ and $\tilde{\mathcal{N}}$ with the respective initial conditions at $t=0$ given by $v_{0}$ and $\left(\mathbf{x}, v_{0}\right)$. Let $r\left(t, \mathbf{x}-\mathbf{y}, v_{0}, w\right)$, $t \in(0,+\infty), \mathbf{x}, \mathbf{y} \in \mathbb{R}^{2}, v_{0}, w \in \mathbb{R}$ be the transition of probability density that corresponds to $\tilde{\mathcal{R}}_{\mathbf{x}, v_{0}}$. Using Corollary 3.25 p. 22 of [6] we have that for some constants $C, m>0$

$$
r\left(t, \mathbf{y}, v_{0}, w\right) \leq C t^{-m}, \quad \forall \mathbf{y} \in \mathbb{R}^{2}, v_{0}, w \in \mathbb{R}, t \in(0,1] .
$$

Denote by $\tau_{Z}(\pi)$ the exit time of a path $\pi \in \mathcal{C}^{1}$ from the set $Z$. For any $\pi \in \mathcal{C}^{3}$ we set also $\tilde{\tau}_{Z}(\pi)=\tau_{Z}(V(\cdot ; \pi))$. Let $S:[-1,1] \rightarrow \mathbb{S}$ be given by $S(v):=\left(v, \sqrt{1-v^{2}}\right), \quad v \in[-1,1]$ and let $\tilde{S}: \mathcal{C}^{3} \rightarrow \mathcal{C}$ be given by $\tilde{S}(\pi)(t):=(X(t ; \pi), S \circ V(t ; \pi)), t \geq 0$. For any $A \in \mathcal{M}^{\tilde{\tau}_{Z}}$ we have $\tilde{\mathcal{R}}_{\mathbf{x}, v_{1}}\left[\tilde{S}^{-1}(A)\right]=\mathfrak{Q}_{\mathbf{x}, \mathbf{v}}[A]$. Since the event

$$
\left[\left|X\left(\frac{1}{p_{1}}\right)-\mathbf{y}\right| \leq \frac{7}{p_{2}}\right] \cap\left[\left|\hat{V}\left(\frac{1}{p_{1}}\right) \cdot \hat{\mathbf{w}}\right| \geq 1-\frac{9}{N_{4}}\right] \cap\left[\tilde{\tau}_{Z} \geq \frac{1}{p_{1}}\right]
$$


is $\mathcal{M}^{\tilde{\tau}_{Z}}$-measurable we have

$$
\begin{aligned}
& \mathfrak{Q}_{\mathbf{x}, \mathbf{v}}\left[\left|X\left(\frac{1}{p_{1}}\right)-\mathbf{y}\right| \leq \frac{7}{p_{2}}, \quad\left|\hat{V}\left(\frac{1}{p_{1}}\right) \cdot \hat{\mathbf{w}}\right| \geq 1-\frac{9}{N_{4}}\right] \\
& \leq \tilde{\mathcal{R}}_{\mathbf{x}, v_{1}}\left[\left|X\left(\frac{1}{p_{1}}\right)-\mathbf{y}\right| \leq \frac{7}{p_{2}}, \quad\left|V\left(\frac{1}{p_{1}}\right)-w_{1}\right| \leq \frac{3 \sqrt{2} v}{N_{4}^{1 / 2}}, \quad \tilde{\tau}_{Z} \geq \frac{1}{p_{1}}\right]+\mathcal{R}_{v_{1}}\left[\tau_{Z}<\frac{1}{p_{1}}\right] \\
& \leq C\left(\frac{p_{1}^{m}}{p_{2}^{2} N_{4}^{1 / 2}}+e^{-C_{3} p_{1}}\right)
\end{aligned}
$$

with $v=|\mathbf{w}|$. To obtain the last inequality we have used (5.22) to bound the first term in the second line, and an elementary estimate for non-degenerate diffusions stating that $\mathcal{R}_{v_{1}}\left[\tau_{Z}<1 / p_{1}\right]<$ $C e^{-C_{3} p_{1}}$ for some constants $C, C_{3}>0$ - see, for instance, (2.1) p. 87 of $[8$. Inequality (5.23) implies (5.20) for $j=1$ with $C_{2}:=m$. To finish the induction argument assume that (5.20) holds for a certain $j$. We show that it holds for $j+1$ with the same constants $C_{1}, C_{2}$ and $C_{3}>0$. The latter follows easily from the Chapman-Kolmogorov equation, since

$$
\begin{aligned}
& \mathfrak{Q}_{\mathbf{x}, \mathbf{v}}\left[\left|X\left(\frac{j+1}{p_{1}}\right)-\mathbf{y}\right| \leq \frac{7}{p_{2}}, \quad\left|\hat{V}\left(\frac{j+1}{p_{1}}\right) \cdot \hat{\mathbf{w}}\right| \geq 1-\frac{9}{N_{4}}\right] \\
& =\iint_{\mathbb{R}^{2} \times \mathbb{S}_{v}} \mathfrak{Q}_{\mathbf{z}, \mathbf{u}}\left[\left|X\left(\frac{j}{p_{1}}\right)-\mathbf{y}\right| \leq \frac{7}{p_{2}}, \quad\left|\hat{V}\left(\frac{j}{p_{1}}\right) \cdot \hat{\mathbf{w}}\right| \geq 1-\frac{9}{N_{4}}\right] Q\left(\frac{1}{p_{1}}, \mathbf{x}, \mathbf{v}, d \mathbf{z}, d \mathbf{u}\right) \\
& \leq C\left(\frac{p_{1}^{m}}{p_{2}^{2} N_{4}^{1 / 2}}+e^{-C_{3} p_{1}}\right) \iint Q\left(\frac{1}{p_{1}}, \mathbf{x}, \mathbf{v}, d \mathbf{y}, d \mathbf{l}\right)=C\left(\frac{p_{1}^{m}}{p_{2}^{2} N_{4}^{1 / 2}}+e^{-C_{3} p_{1}}\right)
\end{aligned}
$$

and the formula (5.20) for $j+1$ follows. We used the induction hypothesis in the last inequality above. We denoted by $Q(t, \mathbf{x}, \mathbf{v}, \cdot, \cdot)$ the transition of probability corresponding to the path measure $\mathfrak{Q}_{\mathbf{x}, \mathbf{v}}$.

We are going to use now Proposition 5.3 and Lemma 5.4 to finish the proof of (5.7). Assume that $\epsilon_{i}, i=1, \ldots, 8$ satisfy the assumptions of Proposition 3.5 and let $\mathbf{w} \in \mathbb{R}_{*}^{2}$ with $|\mathbf{w}|=v$. Suppose that $f_{\mathrm{w}}^{(\delta)}: \mathbb{R}_{*}^{4} \rightarrow[0,1]$ is a $C^{\infty}$-regular function that satisfies $f_{\mathbf{w}}^{(\delta)}(\mathbf{x}, \mathbf{v})=1$, if $|\mathbf{x}| \leq 5 / p_{2}$ and $|\mathbf{v} \cdot \mathbf{w}| \geq\left(1-8 / N_{4}\right) v^{2}$. In addition we assume that $f_{\mathbf{w}}^{(\delta)}(\mathbf{x}, \mathbf{v})=0$, if either $|\mathbf{x}| \geq 6 / p_{2}$, or $|\mathbf{v} \cdot \mathbf{w}| \leq\left(1-9 / N_{4}\right) v^{2}$. We can choose $f_{\mathbf{w}}^{(\delta)}$ so that $\left\|f_{\mathbf{w}}^{(\delta)}\right\|_{3,3} \leq 2\left(N_{4}^{1 / 2} p_{2}\right)^{3}$. For any $\mathbf{x}_{0} \in \mathbb{R}^{d}$ and $i / p_{2} \leq t \leq j / p_{2}$ define

$$
G_{j}\left(t, \mathbf{x}, \mathbf{v} ; \mathbf{x}_{0}, \mathbf{w}\right):=\mathfrak{M}_{\mathbf{x}, \mathbf{v}}\left[f_{\mathbf{w}}^{(\delta)}\left(X\left(\frac{j}{p_{2}}-t\right)-\mathbf{x}_{0}, V\left(\frac{j}{p_{2}}-t\right)\right)\right] .
$$

Obviously, we have

$$
\partial_{t} G_{j}\left(t, \mathbf{x}, \mathbf{v v} ; \mathbf{x}_{0}, \mathbf{w}\right)+\tilde{\mathcal{L}} G_{j}\left(t, \mathbf{x}, \mathbf{v} ; \mathbf{x}_{0}, \mathbf{w}\right)=0 .
$$

Hence, using Proposition 5.3 with $u=j / p_{2}$ and $t=i / p_{2}$, we obtain that

$$
\begin{aligned}
& \left|\tilde{E}_{\mathbf{x}, \mathbf{v}}^{(\delta)}\left[f_{\mathbf{w}}^{(\delta)}\left(X\left(\frac{j}{p_{2}}\right)-\mathbf{x}_{0}, V\left(\frac{j}{p_{2}}\right)\right)-G_{j}\left(\frac{i}{p_{2}}, X\left(\frac{i}{p_{2}}\right), V\left(\frac{i}{p_{2}}\right) ; \mathbf{x}_{0}, \mathbf{w}\right) \mid \mathcal{M}^{i / p_{2}}\right]\right| \\
& \leq C\left\|G_{j}\left(\cdot, \cdot, \cdot ; \mathbf{x}_{0}, \mathbf{w}\right)\right\|_{1,1,3}^{\left[i / p_{2}, j / p_{2}\right]} \delta^{1 / 30}, \quad \forall \delta \in\left(0, \delta_{*}\right] .
\end{aligned}
$$

According to [7] Theorem 2.58, p. 53 we have

$$
\left\|G_{j}\left(\cdot, \cdot, \cdot ; \mathbf{x}_{0}, \mathbf{w}\right)\right\|_{1,1,3}^{\left[i / p_{2}, j / p_{2}\right]} \leq C\left\|f_{\mathbf{w}}^{(\delta)}\right\|_{3,3} \leq C\left(N_{4}^{1 / 2} p_{2}\right)^{3} \leq C \delta^{-3\left(\epsilon_{1}+\epsilon_{2}+\epsilon_{8} / 2\right)}, \quad j \in\left\{0, \ldots,\left[p_{2} T\right]\right\} .
$$


Hence combining (5.24) and (5.25) we obtain that the left hand side of (5.24) is less than, or equal to $C \delta^{1 / 30-3\left(\epsilon_{2}+\epsilon_{3}+\epsilon_{8} / 2\right)}$ for all $\delta \in(0,1]$. Assume now that $u=j / p_{2} \geq t+1 / p_{1}$ with $t=i / p_{2}$ and set $i_{0}=j-\frac{p_{2}}{p_{1}}$, so that $1 \leq i \leq i_{0} \leq j \leq\left[T p_{2}\right]$. We have

$$
\begin{aligned}
& R_{\mathbf{x}, \mathbf{v}}^{(\delta)}\left[\left|X\left(\frac{j}{p_{2}}\right)-X\left(\frac{i}{p_{2}}\right)\right| \leq \frac{5}{p_{2}},\left|\hat{V}\left(\frac{j}{p_{2}}\right) \cdot \hat{V}\left(\frac{i}{p_{2}}\right)\right| \geq 1-\frac{8}{N_{4}}\right] \\
& \leq \tilde{E}_{\mathbf{x}, \mathbf{v}}^{(\delta)}\left[f_{V\left(i / p_{2}\right)}^{(\delta)}\left(X\left(\frac{j}{p_{2}}\right)-X\left(\frac{i}{p_{2}}\right), V\left(\frac{j}{p_{2}}\right)\right)\right] \\
& \quad=E_{\mathbf{x}, \mathbf{v}}^{(\delta)}\left[\left.E_{\mathbf{x}, \mathbf{v}}^{(\delta)}\left[f_{\mathbf{w}}^{(\delta)}\left(X\left(\frac{j}{p_{2}}\right)-\mathbf{y}, V\left(\frac{j}{p_{2}}\right)\right) \mid \mathcal{M}^{i_{0} / p_{2}}\right]\right|_{\mathbf{y}=X\left(i / p_{2}\right), \mathbf{w}=V\left(i / p_{2}\right)}\right] .
\end{aligned}
$$

According to (5.24) and (5.25) we can estimate the utmost right hand side of (5.26) by

$$
\sup \left\{\mathfrak{M}_{\mathbf{x}, \mathbf{v}} f_{\mathbf{w}}^{(\delta)}\left(X\left(\frac{1}{p_{2}}\right)-\mathbf{y}, V\left(\frac{1}{p_{2}}\right)\right):(\mathbf{x}, \mathbf{v}),(\mathbf{y}, \mathbf{w}) \in \mathcal{A}(2)\right\}+C \delta^{1 / 30-3\left(\epsilon_{1}+\epsilon_{2}+\epsilon_{8} / 2\right)} \text {. }
$$

Now, we may use (5.19) and (5.20) to conclude that $R_{\mathbf{x}, \mathbf{v}}^{(\delta)}[A(\delta)]$ can be estimated by

$$
\begin{aligned}
R_{\mathbf{x}, \mathbf{v}}^{(\delta)}[A(\delta)] & \leq C p_{2}^{2}\left[C_{1} \delta^{\left(2-C_{2}\right) \epsilon_{1}+2 \epsilon_{2}+\epsilon_{8} / 2}+\exp \left\{-C_{3} \delta^{-\epsilon_{1}}\right\}\right]+C \delta^{1 / 30-3\left(\epsilon_{1}+\epsilon_{2}+\epsilon_{8} / 2\right)} \\
& \leq C\left[\delta^{\epsilon_{8} / 2-C_{2} \epsilon_{1}}+\delta^{-2 \epsilon_{2}} \exp \left\{-C_{3} \delta^{-\epsilon_{2}}\right\}\right]+C \delta^{1 / 30-3\left(\epsilon_{1}+\epsilon_{2}+\epsilon_{8} / 2\right)} \leq C \delta^{\gamma}
\end{aligned}
$$

for some $\gamma>0$, provided that

$$
\epsilon_{8}>2 C_{2} \epsilon_{1} .
$$

It is here that the fact that the joint process $(X(t), \hat{V}(t))$ is three-dimensional (and hence transitive) comes into play. This is reflected in (5.29) as the requirement that $N_{4}$ has to be large for $R_{\mathbf{x}, \mathbf{v}}^{(\delta)}[A(\delta)]$ to be small. Note that for $\epsilon_{1}, \epsilon_{2}, \epsilon_{8} \in\left(0,10^{-3}\right)$ we have $3\left(\epsilon_{1}+\epsilon_{2}+\epsilon_{8} / 2\right)<1 / 30$. Summing up, we conclude that for $\epsilon_{i} \in\left(1,10^{-3}\right), i \neq 3,4,7, \epsilon_{1}<\epsilon_{8} /\left(2 C_{2}\right), \epsilon_{3} \in(1 / 7,1 / 6), \epsilon_{4} \in(15 / 16,1)$, $\epsilon_{7} \in(1 / 15,1 / 10)$ we obtain estimate (5.7).

\subsection{The estimate of $\tilde{Q}_{\mathrm{x}, \mathrm{v}}^{(\delta)}[B(\delta)]$ and weak convergence of measures.}

It remains to estimate the probability of the event $B(\delta)$ defined in (5.5) in order to finish the proof of Theorem [5.1] Let $\gamma_{0}>0$ and introduce the following stopping time

$$
\sigma_{\delta}(\pi):=\min \left[t: \sup _{0 \leq s<t} \frac{|V(t)-V(s)|}{(t-s)^{1 / 2-\gamma_{0}}} \geq \delta^{-\gamma_{0}}\right] .
$$

Assume, in addition to the hypotheses made about $\epsilon_{i}, i=1, \ldots, 8$ in the previous section that

$$
\epsilon_{*}:=\min \left[\epsilon_{1}-\epsilon_{5}, \epsilon_{1}+\epsilon_{2}-\epsilon_{6}-\epsilon_{8}, \epsilon_{1}+\epsilon_{3}-\epsilon_{7}\right]>0,
$$

that is, that $p_{i} \gg N_{i}$ for $i=1,2,3$. Note that the stopping time $\sigma_{\delta}$ controls all $S_{\delta}^{(j)}, j=1,2,3$ if $\gamma_{0}<\epsilon_{*} / 4$, that is, then

$$
B(\delta) \subseteq B_{1}(\delta),
$$

where $B_{1}(\delta):=\left[\sigma_{\delta} \leq T\right]$, provided that $\delta \in\left(0, \delta_{*}\right]$ and $\delta_{*}$ is sufficiently small. Also, for $\pi \in$ $B(\delta) \backslash A(\delta)$ we have $\sigma_{\delta}(\pi) \leq \tau_{\delta}(\pi)$. 
Suppose that $\tilde{Q}_{*}$ is a weak limit of a sequence $\tilde{Q}_{\mathbf{x}, \mathbf{v}}^{\left(\delta_{n}\right)}$ for some $\delta_{n} \downarrow 0$ and let $\tilde{E}_{*}$ be the corresponding expectation. Let $G \in C_{b}^{1,1,3}\left([0,+\infty), \mathbb{R}_{*}^{4}\right)$ and $N_{t}(G), t \geq 0$ be the process defined by (5.8). Using (5.7) and Proposition 3.5 we conclude that for any fixed $\delta_{0}$ and $G$ as in the statement of Proposition 3.5 the process $N_{t \wedge \sigma_{\delta_{0}}}(G), t \geq 0$ is an $\left(\mathcal{M}^{t}\right)$-martingale under $\tilde{Q}_{*}$. Hence, according to Theorem 6.1 .2 of [8], $\tilde{Q}_{*}$ coincides with $\mathfrak{Q}_{\mathbf{x}, \mathbf{v}}$ on $\mathcal{M}^{\sigma_{\delta_{0}}}$ for an arbitrary $\delta_{0}>0$. Since the set $\left[\sigma_{\delta_{0}} \leq T\right]$ is closed we have, see e.g. Theorem 1.1 .1 of $[$ ]

$$
\limsup _{\delta_{n} \downarrow 0} \tilde{Q}_{\mathbf{x}, \mathbf{v}}^{\left(\delta_{n}\right)}\left[\sigma_{\delta_{0}} \leq T\right] \leq \tilde{Q}_{*}\left[\sigma_{\delta_{0}} \leq T\right]=\mathfrak{Q}_{\mathbf{x}, \mathbf{v}}\left[\sigma_{\delta_{0}} \leq T\right] \leq C \delta_{0}^{\gamma}
$$

for some constants $C, \gamma>0$, see e.g. (2.46) p. 47 of [7]. This proves therefore that $\tilde{Q}_{\mathbf{x}, \mathbf{v}}^{(\delta)}$ converge weakly to $\mathfrak{Q}_{\mathbf{x}, \mathbf{v}}$, as $\delta \downarrow 0$, over $C\left([0, T] ; \mathbb{R}_{*}^{4}\right)$ and

$$
\limsup _{\delta \downarrow 0} \tilde{Q}_{\mathbf{x}, \mathbf{v}}^{(\delta)}\left[\sigma_{\delta_{0}} \leq T\right] \leq C \delta_{0}^{\gamma} .
$$

Moreover, from (5.4) and (5.31) we have

$$
\tilde{Q}_{\mathbf{x}, \mathbf{v}}^{(\delta)}\left[\tau_{\delta} \leq T\right] \leq \tilde{Q}_{\mathbf{x}, \mathbf{v}}^{(\delta)}[A(\delta)]+\tilde{Q}_{\mathbf{x}, \mathbf{v}}^{(\delta)}\left[\sigma_{\delta} \leq T\right] \leq \tilde{Q}_{\mathbf{x}, \mathbf{v}}^{(\delta)}[A(\delta)]+\tilde{Q}_{\mathbf{x}, \mathbf{v}}^{(\delta)}\left[\sigma_{\delta_{0}} \leq T\right]
$$

for $\delta \in\left(0, \delta_{0} \wedge \delta_{*}\right)$, as

$$
\sigma_{\delta^{\prime}} \leq \sigma_{\delta} \quad \text { for } 0<\delta<\delta^{\prime}
$$

Taking the upper limit on both sides of (15.32), as $\delta \downarrow 0$ we obtain that

$$
\limsup _{\delta \downarrow 0} \tilde{Q}_{\mathbf{x}, \mathbf{v}}^{(\delta)}\left[\tau_{\delta} \leq T\right] \leq C \delta_{0}^{\gamma}
$$

Since $\delta_{0}$ was arbitrary we conclude from here that

$$
\lim _{\delta \downarrow} \tilde{Q}_{\mathbf{x}, \mathbf{v}}^{(\delta)}\left[\tau_{\delta} \leq T\right]=0 .
$$

Recall that

$$
\tilde{Q}_{\mathbf{x}, \mathbf{v}}^{(\delta)}\left[A \cap\left[\tau_{\delta}>T\right]\right]=Q_{\mathbf{x}, \mathbf{v}}^{(\delta)}\left[A \cap\left[\tau_{\delta}>T\right]\right]
$$

for all $A \in \mathcal{M}^{T}$. Here, as we recall $Q_{\mathbf{x}, \mathbf{v}}^{(\delta)}$, is the law of the solution to (2.5) without the cut-offs. Thus, we can conclude that in fact $Q_{\mathbf{x}, \mathbf{v}}^{(\delta)}$ converges weakly, as $\delta \downarrow 0$, to $\mathfrak{Q}_{\mathbf{x}, \mathbf{v}}$. This finishes the proof of Theorem 2.1]

\section{A The proof of Lemma 3.2}

The proof of (3.19) - (3.21). Here we also explain why both vectors $V\left(t_{k-1}^{(i)}\right)$ and $V\left(t_{k}^{(i)}\right)$ are used in the definition of stopping time $S_{\delta}^{(i)}$. First, we prove the following statement.

Proposition A.1 For any $i=1,2,3$ we have

$$
\hat{\boldsymbol{l}}^{(\delta)}(t) \cdot \hat{\boldsymbol{l}}^{(\delta)}\left(t_{k-1}^{(i)}\right) \geq 1-\frac{2}{N_{i}}
$$

and

$$
\left|\boldsymbol{l}^{(\delta)}(t)-\boldsymbol{l}^{(\delta)}\left(t_{k}^{(i)}\right)\right| \leq \frac{1}{M_{*} N_{i}^{1 / 2}}
$$

for $t \in\left[t_{k}^{(i)}, t_{k+1}^{(i)}\right)$ and all $k \geq 0$. 
Proof. We show (A.1) by induction. First, let $k=0$. The potential set of bad times on the interval $\left[0, t_{1}^{(i)}\right)$

$$
G_{0}:=\left[t \in\left[0, t_{1}^{(i)}\right):\left|\boldsymbol{l}^{(\delta)}(t)-\boldsymbol{l}^{(\delta)}(0)\right|>\frac{1}{M_{*}}\left(\frac{1}{N_{i}}\right)^{1 / 2}, \text { or } \quad \hat{\boldsymbol{l}}^{(\delta)}(t) \cdot \hat{\boldsymbol{l}}^{(\delta)}(0)<1-\frac{2}{N_{i}}\right]
$$

is open. Note that obviously $0 \in G_{0}^{c}$ so that $G_{0}^{c}$ is not empty. We can find therefore a countable family of disjoint intervals $\left(a_{j}, b_{j}\right), j \geq 1$ such that $G_{0}=\bigcup_{j}\left(a_{j}, b_{j}\right)$ with $a_{j}<b_{j}$. We must have $a_{j} \in G_{0}^{c}$ so both $\left|\boldsymbol{l}^{(\delta)}\left(a_{j}\right)-\boldsymbol{l}^{(\delta)}(0)\right| \leq M_{*}^{-1} N_{i}^{-1 / 2}$ and $\hat{\boldsymbol{l}}^{(\delta)}\left(a_{j}\right) \cdot \hat{\boldsymbol{l}}^{(\delta)}(0) \geq 1-2 / N_{i}$. Using the cut-off condition we deduce that the function $F_{\delta}$ defined in (3.16) vanishes: $F_{\delta}=0$ on the interval $\left(a_{i}, b_{i}\right)$ and therefore

$$
\frac{d \boldsymbol{l}^{(\delta)}}{d t}(t)=0 \quad \text { and } \quad \boldsymbol{l}^{(\delta)}(t)=\boldsymbol{l}^{(\delta)}\left(a_{j}\right) \quad \text { for } t \in\left(a_{j}, b_{j}\right)
$$

so we have both $\left|\hat{\boldsymbol{l}}^{(\delta)}(t)-\hat{\boldsymbol{l}}^{(\delta)}(0)\right| \geq M_{*} N_{i}^{-1 / 2}$ and $\hat{\boldsymbol{l}}^{(\delta)}(t) \cdot \hat{\boldsymbol{l}}^{(\delta)}(0) \geq 1-2 / N_{i}$ for $t \in\left(a_{j}, b_{j}\right)$. This, however, means, by the definition of the set $G_{0}$ that $\left(a_{j}, b_{j}\right) \subset G_{0}^{c}$, which is a contradiction to the way the intervals $\left(a_{j}, b_{j}\right)$ were defined. This shows that in fact $G_{0}$ is empty.

Suppose now that A.1 holds for a certain $k$. Once again, the set

$$
G_{k+1}:=\left[t \in\left[t_{k+1}^{(i)}, t_{k+2}^{(i)}\right): \hat{\boldsymbol{l}}^{(\delta)}(t) \cdot \hat{\boldsymbol{l}}^{(\delta)}\left(t_{k}^{(i)}\right)<1-\frac{2}{N_{i}}, \text { or }\left|\boldsymbol{l}^{(\delta)}(t)-\boldsymbol{l}^{(\delta)}\left(t_{k+1}^{(i)}\right)\right|>\frac{1}{M_{*} N_{i}^{1 / 2}}\right]
$$

is obviously open. It follows from the induction assumption that

$$
\left|\boldsymbol{l}^{(\delta)}\left(t_{k+1}^{(i)}\right)-\boldsymbol{l}^{(\delta)}\left(t_{k}^{(i)}\right)\right| \leq \frac{1}{M_{*} N_{i}^{1 / 2}}
$$

which implies that

$$
\left|\hat{\boldsymbol{l}}^{(\delta)}\left(t_{k+1}^{(i)}\right)-\hat{\boldsymbol{l}}^{(\delta)}\left(t_{k}^{(i)}\right)\right| \leq \frac{2}{N_{i}^{1 / 2}}
$$

since $\left(2 M_{*}\right)^{-1} \leq\left|\boldsymbol{l}^{(\delta)}\left(t_{k+1}^{(i)}\right)\right|,\left|\boldsymbol{l}^{(\delta)}\left(t_{k}^{(i)}\right)\right|$. It follows that $\hat{\boldsymbol{l}}^{(\delta)}\left(t_{k+1}^{(i)}\right) \cdot \hat{\boldsymbol{l}}^{(\delta)}\left(t_{k}^{(i)}\right) \geq 1-2 / N_{i}$. We conclude that $t_{k+1}^{(i)} \in G_{k+1}^{c}$. We can find therefore a countable family of disjoint open intervals $\left(a_{j}, b_{j}\right)$ such that $G_{k+1}=\bigcup_{j}\left(a_{j}, b_{j}\right)$. Since $a_{i} \in G_{k+1}^{c}$ we have

$$
\hat{\boldsymbol{l}}^{(\delta)}\left(a_{j}\right) \cdot \hat{\boldsymbol{l}}^{(\delta)}\left(t_{k+1}^{(i)}\right) \geq 1-\frac{2}{N_{i}} \text { and }\left|\boldsymbol{l}^{(\delta)}\left(a_{j}\right)-\boldsymbol{l}^{(\delta)}\left(t_{k+1}^{(i)}\right)\right| \leq M_{*}^{-1} N_{i}^{-1 / 2} .
$$

Observe that, as before, for $t \in\left(a_{j}, b_{j}\right)$ equality (A.4) holds, hence, in particular

$$
\boldsymbol{l}^{(\delta)}(t) \cdot \boldsymbol{l}^{(\delta)}\left(t_{k+1}^{(i)}\right) \geq 1-2 / N_{i} \text { and }\left|\boldsymbol{l}^{(\delta)}(t)-\boldsymbol{l}^{(\delta)}\left(t_{k+1}^{(i)}\right)\right| \leq M_{*}^{-1} N_{i}^{-1 / 2} \text { for } t \in\left(a_{j}, b_{j}\right) .
$$

It follows that $G_{k+1}$ is empty. Thus, holds for all $t \in\left[t_{k+1}^{(i)}, t_{k+2}^{(i)}\right)$.

Since $\left|\boldsymbol{l}^{(\delta)}(t)-\boldsymbol{l}^{(\delta)}\left(t_{k}^{(i)}\right)\right| \leq M_{*}^{-1} N_{i}^{-1 / 2}$ implies $\hat{\boldsymbol{l}}^{(\delta)}(t) \cdot \hat{\boldsymbol{l}}^{(\delta)}\left(t_{k}^{(i)}\right) \geq 1-2 / N_{i}$, a simple consequence of the above proposition is

$$
\hat{\boldsymbol{l}}^{(\delta)}(t) \cdot \hat{\boldsymbol{l}}^{(\delta)}\left(t_{k-1}^{(i)}\right) \geq 1-\frac{2}{N_{i}} \quad \text { and } \quad \hat{\boldsymbol{l}}^{(\delta)}(t) \cdot \hat{\boldsymbol{l}}^{(\delta)}\left(t_{k}^{(i)}\right) \geq 1-\frac{2}{N_{i}}
$$


for $t \in\left[t_{k}^{(i)}, t_{k+1}^{(i)}\right)$ and all $k \geq 0$. Note also that for $t \in\left[t_{k+1}^{(i)}, t_{k+2}^{(i)}\right)$ we obtain from (A.2) that $\left|\boldsymbol{l}^{(\delta)}(t)-\boldsymbol{l}^{(\delta)}\left(t_{k-1}^{(i)}\right)\right| \leq 3 M_{*}^{-1} N_{i}^{-1 / 2}$, hence

$$
\hat{\boldsymbol{l}}^{(\delta)}(t) \cdot \hat{\boldsymbol{l}}^{(\delta)}\left(t_{k-1}^{(i)}\right) \geq 1-\frac{18}{N_{i}} .
$$

This finishes the proof of (3.19) - 3.20). The formula (3.21) follows immediately from A.1).

The proof of (3.18). Let $t_{0}, s_{0}$ be as in the assumptions of Lemma 3.2. In particular, we have

$$
\left|\boldsymbol{y}^{(\delta)}\left(t_{0}\right)-\boldsymbol{y}^{(\delta)}\left(s_{0}\right)\right| \leq \frac{1}{2 p_{2}}
$$

and

$$
\left|\hat{\boldsymbol{l}}^{(\delta)}\left(t_{0}\right) \cdot \hat{\boldsymbol{l}}^{(\delta)}\left(s_{0}\right)\right| \leq 1-\frac{1}{2 N_{4}}
$$

for some $s_{0} \in\left[t_{j_{2}}^{(2)}, t_{j_{2}+1}^{(2)}\right)$ and $t_{0} \in\left[t_{k_{2}}^{(2)}, t_{k_{2}+1}^{(2)}\right)$. Thanks to (A.6) we also have

$$
\left|\hat{\boldsymbol{l}}^{(\delta)}(t)-\hat{\boldsymbol{l}}^{(\delta)}\left(t_{k_{2}}^{(2)}\right)\right|^{2} \leq \frac{4}{N_{2}} \quad \text { for } t \in\left[t_{k_{2}}^{(2)}, t_{k_{2}+1}^{(2)}\right)
$$

and

$$
\left|\hat{\boldsymbol{l}}^{(\delta)}(s)-\hat{\boldsymbol{l}}^{(\delta)}\left(t_{j_{2}}^{(2)}\right)\right|^{2} \leq \frac{4}{N_{2}} \quad \text { for } s \in\left[t_{j_{2}}^{(2)}, t_{j_{2}+1}^{(2)}\right)
$$

On the other hand, A.7 is equivalent to

$$
\left|\hat{\boldsymbol{l}}^{(\delta)}\left(t_{0}\right) \pm \hat{\boldsymbol{l}}^{(\delta)}\left(s_{0}\right)\right|^{2} \geq \frac{1}{N_{4}}
$$

Using (A.8) - A.10 wet for any $t \in\left[t_{k_{2}}^{(2)}, t_{k_{2}+1}^{(2)}\right), s \in\left[t_{j_{2}}^{(2)}, t_{j_{2}+1}^{(2)}\right)$

$$
\begin{aligned}
& \left|\hat{\boldsymbol{l}}^{(\delta)}(t) \pm \hat{\boldsymbol{l}}^{(\delta)}(s)\right| \geq\left|\hat{\boldsymbol{l}}^{(\delta)}\left(t_{0}\right) \pm \hat{\boldsymbol{l}}^{(\delta)}\left(s_{0}\right)\right|-\left|\hat{\boldsymbol{l}}^{(\delta)}\left(t_{0}\right)-\hat{\boldsymbol{l}}^{(\delta)}\left(t_{k_{2}}^{(2)}\right)\right|-\left|\hat{\boldsymbol{l}}^{(\delta)}(t)-\hat{\boldsymbol{l}}^{(\delta)}\left(t_{k_{2}}^{(2)}\right)\right| \\
& -\left|\hat{\boldsymbol{l}}^{(\delta)}\left(s_{0}\right)-\hat{\boldsymbol{l}}^{(\delta)}\left(t_{j_{2}}^{(2)}\right)\right|-\left|\hat{\boldsymbol{l}}^{(\delta)}(s)-\hat{\boldsymbol{l}}^{(\delta)}\left(t_{j_{2}}^{(2)}\right)\right| \geq \frac{1}{N_{4}^{1 / 2}}-\frac{8}{N_{2}^{1 / 2}} \geq \frac{1}{\left(2 N_{4}\right)^{1 / 2}},
\end{aligned}
$$

since $N_{2} \gg N_{4}$ provided that $\delta \in\left(0, \delta_{*}\right]$ and $\delta_{*}>0$ is sufficiently small - see (B.1). Observe that (A.11) is, in turn, equivalent to

$$
\left|\hat{\boldsymbol{l}}^{(\delta)}(t) \cdot \hat{\boldsymbol{l}}^{(\delta)}(s)\right| \leq 1-\frac{1}{4 N_{4}}
$$

which is nothing but (3.18). The proof of Lemma 3.2 is now complete.

\section{B The proof of the intersection lemma}

We prove now an auxiliary statement that we have used in the proof of Proposition 3.3 - that the particle that enters and leaves during the time $\left[t_{i}^{(2)}, t_{i+1}^{(2)}\right]$ a tube $G_{j}$ (see (3.25) for the definition of this set) does not return to this tube before time $t_{i+1}^{(2)}$.

Lemma B.1 The stopping time $\sigma_{2}$ defined by (3.27) is equal to infinity: $\sigma_{2}=+\infty$, that is, the set in the right side of (3.27) is empty. 
Proof. Suppose, on the contrary that $\sigma_{2}<+\infty$. Then obviously, also $\sigma_{1}<+\infty$. Assume that $\tau_{1}, \tau_{2} \in\left[t_{j}^{(2)}, t_{j+1}^{(2)}\right]$ are such that

$$
\left|\boldsymbol{y}^{(\delta)}\left(\tau_{l}\right)-\boldsymbol{y}^{(\delta)}\left(\sigma_{l}\right)\right|=\min \left[\left|\boldsymbol{y}^{(\delta)}(t)-\boldsymbol{y}^{(\delta)}\left(\sigma_{l}\right)\right|: t \in\left[s_{j+1}^{(2)}, s_{j+1}^{(q)}\right]\right], \quad l=1,2 .
$$

According to (3.21) the oscillation of the unit tangent vector $\hat{\boldsymbol{l}}^{(\delta)}(t)$ to the curve $\Gamma_{j}$ is bounded by $C / N_{2}^{1 / 2}$. Hence, there exists a continuous function $\Phi(t)$ such that for $t \in\left[t_{j}^{(2)}, t_{j+1}^{(2)}\right]$ we have

$$
\hat{\boldsymbol{l}}^{(\delta)}(t)=[\cos \Phi(t), \sin \Phi(t)], \quad t \in\left[t_{j}^{(2)}, t_{j+1}^{(2)}\right]
$$

and, moreover,

$$
\max \left[|\Phi(t)-\Phi(s)|: s, t \in\left[t_{j}^{(2)}, t_{j+1}^{(2)}\right]\right] \leq C / N_{2}^{1 / 2}
$$

for some constant $C>0$.

This fact precludes any possible self-intersections of $\Gamma_{j}$. Indeed, suppose that $s_{0}<t_{0}$ were such that $\boldsymbol{y}^{(\delta)}\left(s_{0}\right)=\boldsymbol{y}^{(\delta)}\left(t_{0}\right)$ and $\boldsymbol{y}^{(\delta)}(s) \neq \boldsymbol{y}^{(\delta)}(t)$ for $s, t \in\left(s_{0}, t_{0}\right)$. Denote by $\Phi_{0} \in[-\pi, \pi)$ the oriented angle between vectors $\hat{\boldsymbol{l}}^{(\delta)}\left(t_{0}\right)$ and $\hat{\boldsymbol{l}}^{(\delta)}\left(s_{0}\right)$ - see Figure B] Again, thanks to (3.21) we obtain that

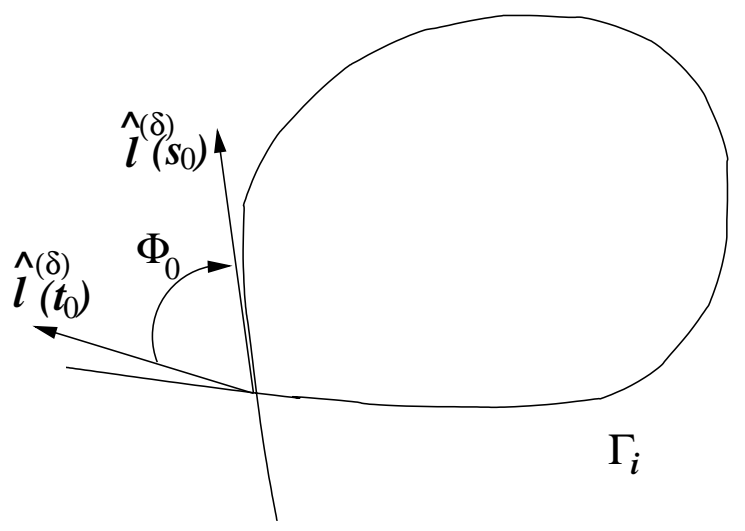

Figure 2

$\left|\Phi_{0}\right| \leq C / N_{2}^{1 / 2}$. Then, according to the Index Theorem, see e.g. Theorem 2.1, p. 147 of [3], we would have $\Phi\left(t_{0}\right)-\Phi\left(s_{0}\right)+\Phi_{0}=2 \pi$, which is impossible for the left hand side of the equality cannot exceed $C / N_{2}^{1 / 2}$. Hence, the curve $\Gamma_{j}$ does not intersect itself - the same argument shows that neither does $\Gamma_{i}$.

We complement the $\operatorname{arc} \Gamma_{j}$ with two half-lines $L_{1}, L_{2}$ that start at points $\boldsymbol{y}^{(\delta)}\left(t_{j}^{(2)}\right)$ and $\boldsymbol{y}^{(\delta)}\left(t_{j+1}^{(2)}\right)$ and run in the directions $-\hat{\boldsymbol{l}}^{(\delta)}\left(t_{j}^{(2)}\right)$ and $\hat{\boldsymbol{l}}^{(\delta)}\left(t_{j+1}^{(2)}\right)$ respectively, see Figure $\mathbf{B}$. We obtain in such a way an unbounded $C^{1}$-curve, we call it $\Gamma$, that cuts the plane $\mathbb{R}^{2}$ into two unbounded components, say $A$ and $B$. We consider two cases: either the segment $\boldsymbol{y}^{(\delta)}(t), t \in\left[\sigma_{1}, \sigma_{2}\right]$ lies in a single component, or not. The latter means that there must be a point $\tilde{\sigma} \in\left[\sigma_{1}, \sigma_{2}\right]$ such that $\boldsymbol{y}^{(\delta)}(\tilde{\sigma}) \in L_{1} \cup L_{2}$. Assume further that $\boldsymbol{y}^{(\delta)}(t), t \in\left(\sigma_{1}, \tilde{\sigma}\right)$ lies entirely in the component $A$, see Figure $\mathbb{B}$. Let $\mathbf{n}_{1}$ and $\tilde{\mathbf{n}}$ be the normals to $\Gamma$, directed inwardly w.r.t. $A$, at $\boldsymbol{y}^{(\delta)}\left(\tau_{1}\right)$ and $\boldsymbol{y}^{(\delta)}(\tilde{\sigma})$ correspondingly. Let $\bar{\theta} \in(0, \pi)$ be the non-oriented angle between $\mathbf{n}_{1}$ and $\tilde{\mathbf{n}}$. Thanks to (3.21) we obtain that $\bar{\theta}<C / N_{2}^{1 / 2}$. Since $\sigma_{1}$ is the exit time of $\Gamma_{i}$ from $G_{j}$ the non-oriented angle that $\hat{\boldsymbol{l}}^{(\delta)}\left(\sigma_{1}\right)$ forms with $\mathbf{n}_{1}$ must satisfy $\theta_{1} \leq \pi / 2$. In fact, thanks to the transversality property expressed in (3.18), it must satisfy $\theta_{1} \leq \pi / 2-C / N_{4}^{1 / 2}$. Likewise, we convince ourselves that the non-oriented angle that $\hat{\boldsymbol{l}}^{(\delta)}(\tilde{\sigma})$ forms with $\tilde{\mathbf{n}}$ must satisfy 


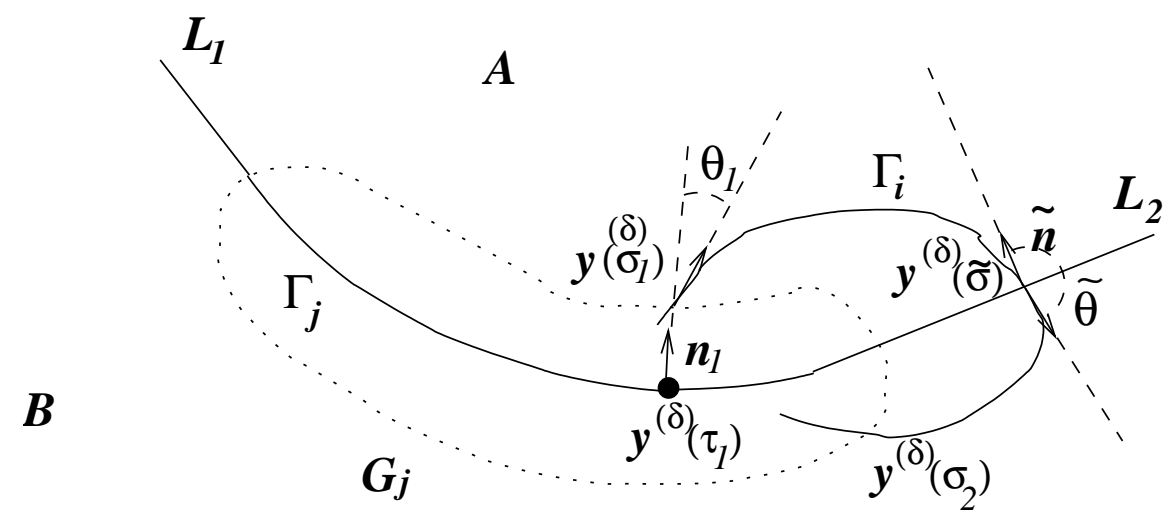

Figure 3.

$\tilde{\theta} \geq \pi / 2+C / N_{4}^{1 / 2}$. The above shows that the change of the argument function $\Phi$ along $\Gamma_{i}$ between $\sigma_{1}$ and $\tilde{\sigma}$ is greater than

$$
\tilde{\theta}-\theta_{1}-\bar{\theta} \geq \frac{C}{N_{4}^{1 / 2}}-\frac{C}{N_{2}^{1 / 2}} \geq \frac{C_{1}}{N_{4}^{1 / 2}}
$$

for a suitable constant $C_{1}>0$ and $\delta \in\left(0, \delta_{*}\right]$, where $\delta_{*}>0$ is sufficiently small as $N_{2} \gg N_{4}$ for small $\delta>0$ - see (3.1). However, (B.1) contradicts (3.21), which proves that $\Gamma_{i}$ may not leave the region $A$.

In the case, when $\boldsymbol{y}^{(\delta)}(t), t \in\left(\sigma_{1}, \sigma_{2}\right)$ lies in $A$, we argue similarly replacing the intersection point $\boldsymbol{y}^{(\delta)}(\tilde{\sigma})$ by $\boldsymbol{y}^{(\delta)}\left(\tau_{2}\right)$ and the normal $\tilde{\mathbf{n}}$ by $\mathbf{n}_{2}$ the normal to $\Gamma$ at $\boldsymbol{y}^{(\delta)}\left(\tau_{2}\right)$ directed into $A$. The remaining part of the argument is virtually identical and leads to the conclusion that $\Gamma_{i}$ may not re-enter the tube $\Gamma_{j}$ and hence $\sigma_{2}=+\infty$.

\section{References}

[1] G. Bal, T. Komorowski and L. Ryzhik, Self-averaging of Wigner Transforms in random media, Comm. Math. Phys., 242, 2003, 81-135.

[2] D. Dürr, S. Goldstein and J. Lebowitz, Asymptotic motion of a classical particle in a random potential in two dimensions: Landau model, Comm. Math. Phys., 113, 1987, 209-230.

[3] P. Hartman, Ordinary Differential Equations, Wiley \& Sons, New York (1964).

[4] H. Kesten, G. C. Papanicolaou, A limit theorem for stochastic acceleration, Comm. Math. Phys., 78, 1980, 19-63.

[5] T. Komorowski and L. Ryzhik, Diffusion in a weakly random Hamiltonian flow, Preprint, 2004. 
[6] S. Kusuoka and D. Stroock, Applications of the Malliavin calculus, Part II, J. Fac. Sci. Univ. Tokyo, Sect. IA, Math, 32, 1985, 1-76.

[7] D. Strook, An Introduction to the Analysis of Paths on a Riemannian Manifold, Math. Surv. and Monographs v. 74, 2000.

[8] D. Strook, S. R. S. Varadhan Multidimensional Diffusion Processes, Berlin, Heidelberg, New York: Springer-Verlag, 1979.

[9] E. Vanden-Eijnden, Some remarks on the quasilinear treatment of the stochastic acceleration problem, Phys. Plasmas, 4, 1997, 1486-1488. 